Draft VERSION NOVEMBER 11, 2018

Preprint typeset using ${ }^{A} \mathrm{~T}_{\mathrm{E}} \mathrm{X}$ style AASTeX6 v. 1.0

\title{
A JANSKY VLA SURVEY OF MAGNETIC CATACLYSMIC VARIABLE STARS: I. THE DATA
}

\author{
PAul E. BArrett ${ }^{1,2}$ AND Christopher Dieck \\ United States Naval Observatory \\ 3450 Massachusetts Ave NW \\ Washington, DC 20392-5420
}

Anthony J. BeAsley

National Radio Astronomical Observatory

520 Edgemont Road

Charlottesville, VA 22903-2475

Kulinder P. Singh

Tata Institute of Fundamental Research

Dr. Homi Bhabha Road, Navy Nagar, Colaba

Mumbai 400005, India

\author{
Paul A. Mason \\ New Mexico State University \\ PO Box 30001 \\ Las Cruces, NM 88003-8001
}

${ }^{1}$ George Washington University

2 paul.barrett@usno.navy.mil

\begin{abstract}
The Jansky Very Large Array was used to observe 121 magnetic cataclysmic variables (MCVs). We report radio detections of 19 stars. Fourteen are new radio sources, increasing the number of MCVs that are radio sources by more than twofold, from 8 to 22 . Most detections are at $8.7 \mathrm{GHz}$ (X-band) with a lesser number at 5.4 and $21.1 \mathrm{GHz}$ (C- and K-bands). Most flux density limits are in the range of $47-470 \mu \mathrm{Jy}$. With the exception of AE Aqr, the maximum flux detected is $818 \mu \mathrm{Jy}$. Fourteen of the detections show approximately $100 \%$ circularly polarized emission, which is characteristic of electron-cyclotron maser emission. The data suggest that MCVs might be divided into two classes of radio emitters: those dominated by weakly polarized gyrosynchrotron emission and those by highly polarized electron-cyclotron maser emission.

Keywords: novae, cataclysmic variables - radio continuum: stars - stars: activity - stars: magnetic fields
\end{abstract}

\section{INTRODUCTION}

Cataclysmic variables $(\mathrm{CVs})$ are binary stars consisting of an accreting white dwarf primary and a lower main sequence, Roche-lobe filling, secondary. They are roughly divided into two classes based on their magnetic properties: systems that have a primary with a strong (>1 MG) magnetic field, the magnetic CVs (MCVs) and those without, the normal or non-magnetic CVs.
The MCVs are further divided into two subclasses; the polars (for polarized stars), characterized by a single optical and X-ray photometric period and strong optical linear (5-10\%) and circular (10-80\%) polarization, and the intermediate polars (IPs) and DQ Her stars, characterized by multiple optical, and possibly X-ray, photometric periods. The magnetic field in the polars is sufficiently strong ( $>10 \mathrm{MG}$ ) to completely disrupt the formation of an accretion disk, whereas the field in 
the IPs and DQ Her stars is inferred to be weaker (1$10 \mathrm{MG}$ ) resulting in a truncated (inner) accretion disk. The IPs are slow rotators with $\mathrm{P}_{\text {spin }}=30-60$ minutes and the three DQ Her systems (DQ Her, AE Aqr, and V533 Her) are fast rotators with $\mathrm{P}_{\text {spin }}=30-60$ seconds, presumably caused by an episode of very high accretion similar to that of the millisecond pulsars.

Studies of CV evolution in the mid-1980s (Rappaport, Verbunt \& Joss 1983, Spruit \& Ritter 1983) showed that the loss of orbital angular momentum due to gravitational radiation at orbital periods $>2 \mathrm{~h}$ was insufficient to drive mass transfer. Another mechanism such as magnetic braking was needed. In this scenario, the outflowing stellar wind from the magnetized secondary star generates a braking torque on the binary and a loss of orbital angular momentum that is sufficiently strong to drive mass transfer at orbital periods between $3-6 \mathrm{~h}$. At $3 \mathrm{~h}$ the secondary star becomes fully convective, which effectively turns off the magnetic braking and mass transfer. At an orbital period of about $2 \mathrm{~h}$, angular momentum loss due to gravitational radiation becomes significant and mass transfer resumes. This evolutionary scenario explains the lack of CVs between two and three hours, which is called the period gap.

For MCVs, the strong magnetic field of the WD extends out to and beyond the secondary. It is therefore possible that some of the mass flow from the secondary flows out of the system along the field lines. At a WD radius of $10^{11} \mathrm{~cm}$, the magnetic field of a $10 \mathrm{MG} W D$ is $\approx 500 \mathrm{G}$, which equates to a cyclotron frequency of 1.4 $\mathrm{GHz}$. Assuming a typical accretion rate of $10^{15} \mathrm{~g} \mathrm{~s}^{-1}$, the radio luminosity is approximately $10^{22} \operatorname{ergs} s^{-1}$. Although this luminosity was well below the sensitivity of the then current radio telescopes, Chanmugan \& Wagner (1978) recommended making radio observations of these systems. Based on this recommendation, R.M. Hjellming (personal communication) observed the polar AM Her (presumably using the Very Large Array), but did not detect it at a sensitivity limit of about $1 \mathrm{mJy}$, where $\mathrm{Jy}=10^{-26} \mathrm{~W} \mathrm{~m}^{-2} \mathrm{~Hz}^{-1}$.

Further observations of AM Her at $4.9 \mathrm{GHz}$ using the Very Large Array (VLA) by Chanmugam \& Dulk (1982) resulted in the first radio detection of a MCV. A flux density of $0.67 \mathrm{mJy}$ was observed. Although the observations were made in full polarization mode, no circular polarization was observed. They concluded that the radio emission from AM Her is due to gyrosynchrotron emission (harmonic number 30-50) from energetic $(\approx 350 \mathrm{keV}$ ) electrons trapped in the magnetosphere of the WD. EF Eri, which was also observed, was not detected at a sensitivity limit of $<0.2 \mathrm{mJy}$. They suggest that the non-detection of EF Eri might be because its magnetic field, which was not known at the time, is considerably different than AM Her; or the or- bital separation of the stars may preclude emission at $4.9 \mathrm{GHz}$. The origin of the energetic electrons was not known, but they suggest several mechanisms for their production, such as shock waves and the unipolar inductor model of Goldreich \& Lynden-Bell (1969).

Subsequent VLA observations of MCVs by Dulk, Bastian \& Chanmugam (1983) again detected radio emission from AM Her at $4.9 \mathrm{GHz}$ and gave upper limits at 1.4 and $15 \mathrm{GHz}$. They also obtained upper limits of about $0.2 \mathrm{mJy}$ at $4.9 \mathrm{GHz}$ for VV Pup, EF Eri, MR Ser, ST LMi, and AN UMa. They suggest that the quiescent emission is the result of energetic electrons $(\approx 500$ $\mathrm{keV}$ ) trapped in the magnetosphere of the WD, provided that the electron energy spectrum is quite hard and the spectral hardness or number density of the energetic electrons increases with radius. Serendipitously, a radio flare lasting about 10 minutes was observed during the observation. The flare had a peak flux of $9.7 \mathrm{mJy}$ $(\approx 20$ times the quiescent emission) and was essentially $100 \%$ circularly polarized. They concluded that the radio emission was due to an electron-cyclotron maser that operates near the surface of the red-dwarf companion in $\mathrm{a} \approx 1 \mathrm{kG}$ magnetic field. Magnetic reconnection events due to the interaction of the magnetic fields of the two stars may be the source of the energetic electrons that generate the maser emission.

The first detection of radio emission from a DQ Her binary was reported by Bookbinder \& Lamb (1987) who detect strong radio emission $(\approx 15 \mathrm{mJy}$ at $4.9 \mathrm{GHz}$ and $\approx 5 \mathrm{mJy}$ at $1.4 \mathrm{GHz}$ ) from AE Aqr using the VLA. No radio emission was observed from five other DQ Her stars and intermediate polars (FO Aqr, AO Psc, BG CMi, TV Col, \& EX Hya). They obtained upper limits of $\approx 0.2 \mathrm{mJy}$ at 1.4 and $4.9 \mathrm{GHz}$. In an attempt to test the unipolar inductor model, the asynchronous polar BY Cam was observed using the VLA (Mason, Fisher, \& Chanmugam, G. 1996). The upper limits obtained for this long duration VLA observation are similar to the detections of the current work. Mason \& Gray (2007), during a survey of $9 \mathrm{MCVs}$ at $8.4 \mathrm{GHz}$, also detected AR UMa at flux density of $\approx 0.6 \mathrm{mJy}$. AR UMa has the highest known magnetic field of all polars at 230 MG. The flux densities of two detections were 422 and $734 \mu \mathrm{Jy}$. AM Her was also detected at a flux density of $584 \mu \mathrm{Jy}$. The other seven targets (LW Cam, DO Dra, FIRST J1023.8+0038, SDSS J1553+5516, V2301 Oph, RX J1846.9+5538, and WZ Sge) were not not detected at a flux limit of $\approx 120 \mu \mathrm{Jy}$.

Bastian (1987) observed 15 MCVs, 8 polars and 7 intermediate polars and a DQ Her star, using the VLA. No detections were made at $1.5,4.9$, and $15 \mathrm{GHz}$. Beasley et al. (1994) used the Very Large Array and the Australian Telescope Compact Array to observe an additional 22 MCVs without success. However, Wright et 
al. (1988) reported a detection of the polar V834 Cen at 8.4 GHz using the Parkes $64 \mathrm{~m}$ telescope, and Pavelin, et al. (1994), observing 21 polars and IPs using the Jodrell Bank broadband interferometer, reported detections of five MCVs (BG CMi, ST LMi, DQ Her, AM Her, AE Aqr), three of which were new. For a review of the early radio observations of CVs of all types and a discussion of the radio mechanisms see Chanmugam (1987).

Since those early observations, there has been little interest in observing MCVs in the radio and hence, little progress on understanding the location and mechanism of the radio emission, because the few surveys that were done had little success. However, during the last five years, the much greater sensitivity of the Jansky Very Large Array (JVLA) has significantly improved the situation. Coppejans et al. (2015) and Coppejans et al. (2016) detected radio emission from dwarf nova and nova-like CVs at the tens of $\mu \mathrm{Jy}$ level. For the first survey of nova-like CVs, they observed four targets and detected three of them (RW Sex, V603 Aql, and TT Ari) at $6 \mathrm{GHz}$. For the second survey of dwarf novae, they observed five targets (Z Cam, RX And, SU UMa, YZ Cnc, and $U$ Gem) and detected all of them at flux densities between 12 and $58 \mu \mathrm{Jy}$. The observed radio emission was variable on timescales of minutes to days, which is consistent with previous radio observations of other CVs.

Beginning in 2013, a project was initiated using the Very Long Baseline Array (VLBA) to perform astrometry of radio bright ( $>1 \mathrm{mJy}$ ) MCVs in an attempt to accurately measure their distances. These results will be presented in a separate paper. At the same time, a survey was begun using the JVLA to identify any new radio bright MCVs for follow-up astrometry. The results of that survey are presented here and in a subsequent paper. The current paper presents the results of the data analysis and briefly discusses some observational statistics of the survey, while the second paper presents a more detailed discussion of the data.

\section{OBSERVATIONS AND DATA REDUCTION}

We used the JVLA to observe 121 MCVs during two observing semesters, $13 \mathrm{~B}$ and $15 \mathrm{~A}$, at primarily three frequencies (C-, X-, \& K-bands; 4-6, 8-10 \& 18-22 $\mathrm{GHz}$, respectively) at full polarization. A fourth frequency, Q-band (40-44 GHz), was also used for a few observations. Accurate flux densities were obtained by observing one of four standard flux calibrators (3C48, 3C138, 3C147, and 3C286) during each scheduling block (SB), except for six SBs during semester 13B that used 3C295 as a flux calibrator and could not be accurately flux calibrated by the CASA calibration pipeline. The flux calibrators also served as linear polarization standards. However, accurate linear polarization measurements were not possible, because no polarization leakage calibrators were observed. This omission is not expected to signifanctly impact the measurement of circular polarization. For semester 13B, $40 \mathrm{~h}$ of observing time was requested to observe 60 of the optically brightest, and likely closest, MCVs. The observing program allowed about three MCVs to be observed in each one hour SB. Exposures were approximately two minutes per frequency. Each SB was scheduled twice to increase the probability of detecting the MCVs. The JVLA scheduled 35 of the $40 \mathrm{~h}$, resulting in $42 \mathrm{MCVs}$ being observed. For semester 15A, $69 \mathrm{~h}$ of observing time was requested to observe $69 \mathrm{MCV}$. Except for one SB, each SB contained two MCVs with each exposure being approximately five minutes per frequency. The JVLA scheduled all $69 \mathrm{~h}$ resulting in an additional $69 \mathrm{MCVs}$ being observed. These observations are summarized in Table 1. Column 1 gives the General Catalog of Variable Stars (GCVS) name. If no GCVS name is available, the star's abbreviated constellation name followed by a number is used. Column 2 is the JVLA semester-scheduling-block designation. Column 3 is the date and columns $4-9$ are the UT start times and exposure lengths for the C-, X-, and K-bands, respectively.

Table 1. Log of JVLA observations

\begin{tabular}{lcccccccc}
\hline \multirow{2}{*}{ GVCS Name } & \multirow{2}{*}{ Semester } & \multicolumn{4}{c}{ C-Band } & \multicolumn{2}{c}{ X-Band } & \multicolumn{2}{c}{ K-Band } \\
& & Date & Start & Expo & Start & Expo & Start & Expo \\
& & UT & UT & (s) & UT & (s) & UT & $(\mathrm{s})$ \\
\hline FL Cet & 13B-02A & $2013-09-11$ & $11: 38: 50$ & 110 & $11: 31: 21$ & 111 & $11: 35: 03$ & 111 \\
V1294 Tau & 13B-02A & $2013-09-11$ & $11: 52: 25$ & 115 & $11: 44: 09$ & 120 & $11: 48: 18$ & 120 \\
V1309 Ori & 13B-02A & $2013-09-11$ & $12: 04: 20$ & 110 & $11: 56: 51$ & 108 & $12: 00: 36$ & 111 \\
V709 Cas & 13B-01A & $2013-09-12$ & $11: 13: 05$ & 105 & $11: 09: 36$ & 105 & $11: 06: 06$ & 102 \\
& & & & & & & & \\
\hline
\end{tabular}


Table 1 (continued)

\begin{tabular}{|c|c|c|c|c|c|c|c|c|}
\hline \multirow[t]{2}{*}{ GVCS Name } & \multirow[t]{2}{*}{ Semester } & \multirow[b]{2}{*}{$\begin{array}{c}\text { Date } \\
\text { UT }\end{array}$} & \multicolumn{2}{|c|}{ C-Band } & \multicolumn{2}{|c|}{ X-Band } & \multicolumn{2}{|c|}{ K-Band } \\
\hline & & & $\begin{array}{c}\text { Start } \\
\mathrm{UT}\end{array}$ & $\begin{array}{c}\text { Expo } \\
(\mathrm{s})\end{array}$ & $\begin{array}{c}\text { Start } \\
\mathrm{UT}\end{array}$ & $\begin{array}{c}\text { Expo } \\
(\mathrm{s}) \\
\end{array}$ & $\begin{array}{c}\text { Start } \\
\text { UT }\end{array}$ & $\begin{array}{c}\text { Expo } \\
(\mathrm{s})\end{array}$ \\
\hline Cas 1 & $13 \mathrm{~B}-01 \mathrm{~A}$ & 2013-09-12 & 11:02:05 & 90 & $10: 58: 48$ & 96 & $10: 55: 27$ & 93 \\
\hline BY Cam & 13B-01A & 2013-09-12 & $11: 25: 15$ & 105 & $11: 21: 42$ & 105 & $11: 18: 15$ & 105 \\
\hline V2301 Oph & $13 \mathrm{~B}-16 \mathrm{~A}$ & 2013-09-13 & 03:52:00 & 135 & $03: 44: 21$ & 135 & $03: 36: 42$ & 135 \\
\hline V884 Her & 13B-16A & 2013-09-13 & $03: 31: 55$ & 130 & $03: 27: 33$ & 132 & $03: 23: 12$ & 135 \\
\hline V426 Oph & 13B-16A & 2013-09-13 & $03: 55: 20$ & 135 & $03: 47: 39$ & 135 & 03:40:00 & 135 \\
\hline V603 Aql & $13 \mathrm{~B}-17 \mathrm{~A}$ & 2013-09-13 & $04: 42: 50$ & 110 & $04: 38: 51$ & 114 & $04: 34: 51$ & 114 \\
\hline V373 Sct & 13B-17A & 2013-09-13 & $04: 55: 30$ & 115 & 04:51:30 & 114 & $04: 47: 30$ & 114 \\
\hline V1432 Aql & 13B-17A & 2013-09-13 & 05:00:10 & 115 & 05:04:06 & 114 & 05:08:06 & 114 \\
\hline V552 Aur & 13B-07A & 2013-09-14 & $14: 56: 30$ & 110 & $14: 51: 30$ & 114 & $14: 46: 33$ & 114 \\
\hline SW Uma & 13B-07A & 2013-09-14 & $15: 09: 50$ & 115 & $15: 05: 48$ & 114 & $15: 01: 51$ & 114 \\
\hline DO Dra & 13B-07A & 2013-09-14 & $15: 22: 45$ & 110 & $15: 18: 48$ & 117 & $15: 14: 48$ & 114 \\
\hline BG Cmi & 13B-09A & 2013-09-14 & $13: 25: 00$ & 120 & 13:21:09 & 114 & $13: 17: 21$ & 117 \\
\hline PQ Gem & 13B-09A & 2013-09-14 & $13: 37: 10$ & 115 & $13: 33: 12$ & 120 & $13: 29: 18$ & 120 \\
\hline Hya 1 & 13B-09A & 2013-09-14 & 13:50:10 & 115 & $13: 46: 21$ & 117 & $13: 42: 27$ & 120 \\
\hline AI Tri & $13 \mathrm{~B}-03 \mathrm{~A}$ & 2013-09-15 & $14: 11: 25$ & 105 & $14: 15: 03$ & 105 & $14: 18: 42$ & 102 \\
\hline TT Ari & 13B-03A & 2013-09-15 & $14: 06: 25$ & 125 & $14: 02: 18$ & 129 & $13: 58: 15$ & 129 \\
\hline GK Per & $13 \mathrm{~B}-03 \mathrm{~A}$ & 2013-09-15 & $14: 31: 55$ & 125 & $14: 27: 54$ & 123 & $14: 23: 54$ & 126 \\
\hline FL Cet & 13B-02B & 2013-09-16 & 09:02:45 & 110 & $08: 55: 15$ & 111 & $08: 58: 57$ & 111 \\
\hline V1294 Tau & 13B-02B & 2013-09-16 & 09:16:20 & 115 & 09:08:03 & 120 & 09:12:12 & 120 \\
\hline V1309 Ori & 13B-02B & 2013-09-16 & $09: 28: 15$ & 110 & $09: 20: 45$ & 108 & 09:24:30 & 111 \\
\hline V603 Aql & 13B-17B & 2013-09-16 & $02: 46: 25$ & 110 & $02: 42: 27$ & 114 & $02: 38: 27$ & 114 \\
\hline V373 Sct & 13B-17B & 2013-09-16 & 02:59:05 & 115 & $02: 55: 06$ & 117 & 02:51:06 & 114 \\
\hline V1432 Aql & 13B-17B & 2013-09-16 & 03:03:45 & 115 & 03:07:42 & 114 & 03:11:42 & 114 \\
\hline BY Cam & $13 \mathrm{~B}-06 \mathrm{~A}$ & 2013-09-17 & $11: 15: 10$ & 115 & 11:11:09 & 117 & 11:07:09 & 117 \\
\hline V405 Aur & $13 \mathrm{~B}-06 \mathrm{~A}$ & 2013-09-17 & $11: 27: 05$ & 115 & $11: 23: 06$ & 117 & 11:19:06 & 117 \\
\hline MU Cam & $13 \mathrm{~B}-06 \mathrm{~A}$ & 2013-09-17 & $11: 39: 35$ & 115 & $11: 35: 33$ & 117 & $11: 31: 36$ & 114 \\
\hline BY Cam & 13B-06B & 2013-09-29 & $08: 23: 25$ & 115 & $08: 19: 21$ & 117 & $08: 15: 24$ & 117 \\
\hline V405 Aur & 13B-06B & 2013-09-29 & $08: 35: 20$ & 115 & & & $08: 27: 21$ & 117 \\
\hline MU Cam & 13B-06B & 2013-09-29 & & & $08: 43: 48$ & 117 & 08:39:48 & 117 \\
\hline V2301 Oph & 13B-16B & 2013-09-29 & 03:14:00 & 135 & 03:06:21 & 138 & $02: 58: 42$ & 138 \\
\hline V884 Her & 13B-16B & 2013-09-29 & $02: 53: 55$ & 135 & $02: 49: 33$ & 138 & $02: 45: 15$ & 135 \\
\hline V426 Oph & 13B-16B & 2013-09-29 & $03: 17: 20$ & 135 & 03:09:39 & 138 & 03:02:03 & 135 \\
\hline V709 Cas & 13B-01B & 2013-10-01 & 09:58:20 & 105 & 09:54:51 & 105 & 09:51:21 & 105 \\
\hline Cas 1 & 13B-01B & 2013-10-01 & $09: 47: 20$ & 95 & 09:44:00 & 99 & 09:40:42 & 96 \\
\hline BY Cam & 13B-01B & 2013-10-01 & 10:10:30 & 105 & $10: 06: 57$ & 108 & $10: 03: 27$ & 108 \\
\hline AI Tri & 13B-03B & 2013-10-03 & 07:01:35 & 105 & $07: 05: 12$ & 108 & $07: 08: 54$ & 105 \\
\hline TT Ari & 13B-03B & 2013-10-03 & $06: 56: 35$ & 125 & $06: 52: 30$ & 132 & $06: 48: 27$ & 132 \\
\hline GK Per & 13B-03B & 2013-10-03 & 07:22:05 & 125 & 07:18:06 & 126 & 07:14:06 & 129 \\
\hline QQ Vul & $13 \mathrm{~B}-18 \mathrm{~A}$ & 2013-10-20 & $01: 21: 15$ & 95 & 01:17:03 & 96 & $01: 12: 54$ & 96 \\
\hline WZ Sge & 13B-18A & 2013-10-20 & $01: 25: 35$ & 95 & $01: 33: 51$ & 96 & $01: 29: 42$ & 96 \\
\hline LS Peg & $13 \mathrm{~B}-18 \mathrm{~A}$ & 2013-10-20 & $01: 47: 20$ & 95 & 01:43:39 & 96 & 01:40:00 & 96 \\
\hline
\end{tabular}


Table 1 (continued)

\begin{tabular}{|c|c|c|c|c|c|c|c|c|}
\hline \multirow[t]{2}{*}{ GVCS Name } & \multirow[t]{2}{*}{ Semester } & \multicolumn{3}{|c|}{ C-Band } & \multicolumn{2}{|c|}{ X-Band } & \multicolumn{2}{|c|}{ K-Band } \\
\hline & & $\begin{array}{c}\text { Date } \\
\text { UT }\end{array}$ & $\begin{array}{c}\text { Start } \\
\text { UT }\end{array}$ & $\begin{array}{c}\text { Expo } \\
\text { (s) }\end{array}$ & $\begin{array}{c}\text { Start } \\
\text { UT }\end{array}$ & $\begin{array}{c}\text { Expo } \\
(\mathrm{s})\end{array}$ & $\begin{array}{c}\text { Start } \\
\text { UT }\end{array}$ & $\begin{array}{c}\text { Expo } \\
(\mathrm{s})\end{array}$ \\
\hline GI Mon & 13B-08A & 2013-10-22 & $12: 55: 20$ & 120 & $12: 51: 24$ & 120 & $12: 47: 27$ & 123 \\
\hline VV Pup & $13 \mathrm{~B}-08 \mathrm{~A}$ & 2013-10-22 & $13: 07: 45$ & 120 & 13:03:51 & 120 & $12: 59: 57$ & 120 \\
\hline Hya 2 & $13 \mathrm{~B}-08 \mathrm{~A}$ & 2013-10-22 & $13: 20: 25$ & 115 & $13: 16: 33$ & 117 & $13: 12: 45$ & 117 \\
\hline BG Cmi & 13B-09B & 2013-10-24 & $12: 47: 25$ & 120 & $12: 43: 33$ & 117 & $12: 39: 45$ & 117 \\
\hline PQ Gem & 13B-09B & $2013-10-24$ & $12: 59: 30$ & 120 & $12: 55: 36$ & 123 & $12: 51: 42$ & 123 \\
\hline Hya 1 & 13B-09B & 2013-10-24 & $13: 12: 35$ & 115 & $13: 08: 45$ & 117 & 13:04:51 & 120 \\
\hline GG Leo & $13 \mathrm{~B}-10 \mathrm{~A}$ & $2013-10-24$ & $13: 46: 05$ & 100 & $13: 42: 39$ & 102 & 13:39:09 & 102 \\
\hline ST Lmi & 13B-10A & 2013-10-24 & 14:02:55 & 100 & $13: 56: 57$ & 102 & $13: 50: 57$ & 102 \\
\hline DP Leo & $13 \mathrm{~B}-10 \mathrm{~A}$ & 2013-10-24 & $14: 05: 30$ & 100 & $13: 59: 33$ & 99 & $13: 53: 33$ & 102 \\
\hline EU Uma & $13 \mathrm{~B}-10 \mathrm{~A}$ & $2013-10-24$ & $14: 15: 45$ & 100 & $14: 12: 21$ & 99 & 14:08:54 & 102 \\
\hline GG Leo & 13B-10B & $2013-10-25$ & $14: 12: 05$ & 100 & 14:08:42 & 99 & 14:05:09 & 102 \\
\hline ST Lmi & 13B-10B & 2013-10-25 & 14:29:00 & 100 & $14: 22: 57$ & 102 & $14: 17: 00$ & 102 \\
\hline DP Leo & 13B-10B & $2013-10-25$ & $14: 31: 35$ & 100 & $14: 25: 33$ & 102 & $14: 19: 33$ & 102 \\
\hline EU Uma & 13B-10B & 2013-10-25 & $14: 41: 45$ & 100 & $14: 38: 21$ & 102 & $14: 34: 57$ & 102 \\
\hline V552 Aur & 13B-07B & 2013-10-26 & $13: 56: 15$ & 115 & $13: 51: 15$ & 117 & $13: 46: 15$ & 117 \\
\hline SW Uma & 13B-07B & $2013-10-26$ & 14:09:35 & 115 & $14: 05: 33$ & 117 & $14: 01: 33$ & 117 \\
\hline DO Dra & 13B-07B & $2013-10-26$ & $14: 22: 30$ & 115 & $14: 18: 30$ & 117 & $14: 14: 30$ & 117 \\
\hline GI Mon & 13B-08B & 2013-10-26 & $12: 54: 40$ & 120 & $12: 50: 45$ & 120 & $12: 46: 51$ & 120 \\
\hline VV Pup & 13B-08B & $2013-10-26$ & 13:07:05 & 120 & 13:03:12 & 123 & $12: 59: 18$ & 120 \\
\hline Hya 2 & 13B-08B & $2013-10-26$ & $13: 19: 45$ & 115 & $13: 15: 54$ & 117 & $13: 12: 06$ & 117 \\
\hline TV Col & $13 \mathrm{~B}-05 \mathrm{~A}$ & 2013-10-27 & $13: 07: 20$ & 125 & 13:03:03 & 132 & $12: 58: 48$ & 132 \\
\hline V348 Pup & $13 \mathrm{~B}-05 \mathrm{~A}$ & $2013-10-27$ & $13: 11: 50$ & 125 & $13: 15: 57$ & 126 & $13: 20: 06$ & 126 \\
\hline V351 Pup & $13 \mathrm{~B}-05 \mathrm{~A}$ & 2013-10-27 & $13: 32: 55$ & 125 & $13: 28: 45$ & 126 & $13: 24: 36$ & 126 \\
\hline AN Uma & $13 \mathrm{~B}-11 \mathrm{~A}$ & 2013-10-27 & $14: 14: 20$ & 135 & $14: 05: 48$ & 138 & $13: 57: 21$ & 135 \\
\hline AR Uma & $13 \mathrm{~B}-11 \mathrm{~A}$ & $2013-10-27$ & $14: 18: 10$ & 135 & 14:09:39 & 135 & 14:01:09 & 138 \\
\hline EV Uma & $13 \mathrm{~B}-11 \mathrm{~A}$ & 2013-10-27 & $14: 32: 40$ & 135 & $14: 28: 00$ & 138 & $14: 23: 21$ & 138 \\
\hline HU Aqr & 13B-19A & 2013-10-27 & $02: 37: 25$ & 125 & $02: 33: 15$ & 126 & 02:29:06 & 126 \\
\hline FO Aqr & 13B-19A & $2013-10-27$ & 02:50:35 & 125 & $02: 46: 24$ & 126 & $02: 42: 12$ & 129 \\
\hline $\mathrm{AO}$ Psc & 13B-19A & 2013-10-27 & 03:03:20 & 125 & $02: 59: 12$ & 126 & 02:55:00 & 129 \\
\hline TV Col & 13B-05B & 2013-10-28 & $12: 58: 25$ & 120 & $12: 54: 06$ & 132 & $12: 49: 54$ & 132 \\
\hline V348 Pup & 13B-05B & $2013-10-28$ & $13: 02: 50$ & 125 & 13:07:00 & 126 & 13:11:09 & 126 \\
\hline V351 Pup & 13B-05B & 2013-10-28 & $13: 24: 00$ & 125 & $13: 19: 48$ & 126 & $13: 15: 39$ & 126 \\
\hline AN Uma & 13B-11B & 2013-10-28 & $14: 05: 25$ & 130 & $13: 56: 54$ & 135 & $13: 48: 24$ & 138 \\
\hline AR Uma & 13B-11B & $2013-10-28$ & 14:09:10 & 135 & 14:00:42 & 138 & $13: 52: 15$ & 135 \\
\hline EV Uma & 13B-11B & $2013-10-28$ & $14: 23: 45$ & 135 & 14:19:03 & 138 & $14: 14: 24$ & 138 \\
\hline QQ Vul & 13B-18B & 2013-11-03 & $03: 39: 25$ & 95 & $03: 35: 15$ & 96 & 03:31:06 & 96 \\
\hline WZ Sge & $13 \mathrm{~B}-18 \mathrm{~B}$ & 2013-11-03 & $03: 43: 45$ & 95 & $03: 52: 03$ & 96 & $03: 47: 54$ & 96 \\
\hline LS Peg & 13B-18B & 2013-11-03 & 04:05:30 & 95 & 04:01:51 & 96 & $03: 58: 12$ & 96 \\
\hline HU Aqr & 13B-19B & 2013-11-06 & $03: 42: 50$ & 125 & 03:38:39 & 126 & 03:34:30 & 126 \\
\hline FO Aqr & 13B-19B & 2013-11-06 & $03: 55: 55$ & 125 & $03: 51: 45$ & 129 & $03: 47: 36$ & 129 \\
\hline $\mathrm{AO}$ Psc & 13B-19B & 2013-11-06 & 04:08:45 & 125 & 04:04:33 & 129 & 04:00:24 & 129 \\
\hline
\end{tabular}


Table 1 (continued)

\begin{tabular}{|c|c|c|c|c|c|c|c|c|}
\hline \multirow[t]{2}{*}{ GVCS Name } & \multirow[t]{2}{*}{ Semester } & \multirow[b]{2}{*}{$\begin{array}{c}\text { Date } \\
\text { UT }\end{array}$} & \multicolumn{2}{|c|}{ C-Band } & \multicolumn{2}{|c|}{ X-Band } & \multicolumn{2}{|c|}{ K-Band } \\
\hline & & & $\begin{array}{c}\text { Start } \\
\text { UT }\end{array}$ & $\begin{array}{c}\text { Expo } \\
\text { (s) }\end{array}$ & $\begin{array}{c}\text { Start } \\
\text { UT }\end{array}$ & $\begin{array}{c}\text { Expo } \\
\text { (s) }\end{array}$ & $\begin{array}{c}\text { Start } \\
\text { UT }\end{array}$ & $\begin{array}{c}\text { Expo } \\
\text { (s) }\end{array}$ \\
\hline HU Aqr & 13B-19B & 2013-11-07 & $00: 25: 25$ & 75 & $00: 22: 15$ & 78 & 00:19:06 & 78 \\
\hline FO Aqr & 13B-19B & 2013-11-07 & $00: 38: 10$ & 75 & $00: 34: 57$ & 78 & $00: 31: 48$ & 78 \\
\hline AO Psc & 13B-19B & 2013-11-07 & $00: 50: 50$ & 75 & $00: 47: 42$ & 75 & $00: 44: 33$ & 75 \\
\hline V795 Her & 13B-15A & 2013-12-02 & $15: 53: 45$ & 125 & $15: 49: 36$ & 126 & $15: 45: 27$ & 126 \\
\hline DQ Her & 13B-15A & 2013-12-02 & $16: 06: 45$ & 125 & $16: 02: 33$ & 126 & $15: 58: 24$ & 126 \\
\hline AM Her & 13B-15A & 2013-12-02 & $16: 10: 55$ & 120 & $16: 15: 00$ & 129 & 16:19:09 & 129 \\
\hline V795 Her & 13B-15B & 2014-01-06 & $14: 26: 45$ & 125 & $14: 22: 36$ & 126 & $14: 18: 27$ & 126 \\
\hline DQ Her & 13B-15B & 2014-01-06 & $14: 39: 45$ & 125 & $14: 35: 33$ & 126 & $14: 31: 24$ & 126 \\
\hline AM Her & 13B-15B & 2014-01-06 & $14: 43: 55$ & 120 & 14:48:00 & 129 & 14:52:09 & 129 \\
\hline $\mathrm{BM} \mathrm{CrB}$ & 13B-13A & 2014-01-17 & $13: 23: 25$ & 125 & $13: 19: 12$ & 129 & $13: 15: 03$ & 129 \\
\hline MR Ser & 13B-13A & 2014-01-17 & $13: 36: 50$ & 125 & $13: 32: 42$ & 126 & $13: 28: 33$ & 126 \\
\hline V519 Ser & 13B-13A & 2014-01-17 & $13: 49: 20$ & 125 & 13:45:09 & 126 & 13:41:00 & 126 \\
\hline EF Eri & 13B-04A & 2014-01-18 & $04: 23: 40$ & 85 & 04:20:30 & 87 & $04: 17: 21$ & 87 \\
\hline VY For & 13B-04A & 2014-01-18 & $04: 27: 10$ & 85 & 04:30:18 & 87 & $04: 33: 27$ & 87 \\
\hline AH Eri & 13B-04A & 2014-01-18 & 04:48:05 & 85 & $04: 42: 36$ & 87 & 04:37:09 & 87 \\
\hline IW Eri & 13B-04A & 2014-01-18 & $04: 50: 25$ & 85 & $04: 44: 57$ & 87 & $04: 39: 27$ & 87 \\
\hline EF Eri & 13B-04B & 2014-02-10 & 02:38:30 & 85 & $02: 35: 18$ & 87 & 02:32:09 & 87 \\
\hline VY For & 13B-04B & 2014-02-10 & 02:42:00 & 80 & 02:45:06 & 87 & $02: 48: 15$ & 87 \\
\hline AH Eri & 13B-04B & 2014-02-10 & 03:02:55 & 85 & $02: 57: 24$ & 87 & $02: 51: 54$ & 87 \\
\hline IW Eri & 13B-04B & 2014-02-10 & 03:05:15 & 85 & 02:59:45 & 84 & $02: 54: 15$ & 87 \\
\hline BS Tri & $15 \mathrm{~A}-02 \mathrm{~A}$ & 2015-03-05 & $03: 01: 25$ & 290 & $02: 47: 54$ & 291 & 02:54:39 & 291 \\
\hline XY Ari & $15 \mathrm{~A}-02 \mathrm{~A}$ & 2015-03-05 & 03:08:40 & 285 & $03: 21: 45$ & 291 & $03: 15: 12$ & 291 \\
\hline Lyn 1 & $15 \mathrm{~A}-14 \mathrm{~A}$ & 2015-03-06 & 00:51:05 & 280 & 00:38:00 & 282 & 00:44:30 & 285 \\
\hline Cnc 2 & $15 \mathrm{~A}-14 \mathrm{~A}$ & 2015-03-06 & 00:58:10 & 280 & 01:11:03 & 282 & 01:04:36 & 285 \\
\hline LS Cam & $15 \mathrm{~A}-08 \mathrm{~A}$ & 2015-03-07 & 09:57:05 & 305 & 09:43:30 & 300 & 09:50:15 & 306 \\
\hline HS Cam & $15 \mathrm{~A}-08 \mathrm{~A}$ & 2015-03-07 & 10:03:50 & 305 & $10: 17: 30$ & 300 & 10:10:39 & 306 \\
\hline FH Uma & $15 \mathrm{~A}-21 \mathrm{~A}$ & 2015-03-07 & 10:47:00 & 265 & $10: 34: 42$ & 267 & $10: 40: 51$ & 267 \\
\hline EK Uma & $15 \mathrm{~A}-21 \mathrm{~A}$ & 2015-03-07 & 10:53:30 & 265 & $11: 05: 48$ & 264 & 10:59:39 & 264 \\
\hline V4738 Sgr & $15 \mathrm{~A}-35 \mathrm{~A}$ & 2015-03-07 & $15: 53: 40$ & 115 & $15: 46: 33$ & 114 & $15: 50: 03$ & 117 \\
\hline LW Cam & $15 \mathrm{~A}-10 \mathrm{~A}$ & 2015-03-08 & 09:10:15 & 305 & 08:56:51 & 300 & 09:03:30 & 306 \\
\hline HT Cam & $15 \mathrm{~A}-10 \mathrm{~A}$ & 2015-03-08 & 09:17:30 & 305 & 09:31:24 & 303 & $09: 24: 27$ & 306 \\
\hline HY Leo & $15 \mathrm{~A}-20 \mathrm{~A}$ & 2015-03-08 & 10:01:10 & 290 & 09:48:09 & 285 & $09: 54: 36$ & 294 \\
\hline Leo 1 & $15 \mathrm{~A}-20 \mathrm{~A}$ & 2015-03-08 & 10:06:55 & 290 & 10:20:03 & 285 & 10:13:30 & 291 \\
\hline Sct 1 & $15 \mathrm{~A}-27 \mathrm{~A}$ & 2015-03-08 & $11: 11: 25$ & 290 & $10: 58: 27$ & 291 & 11:04:57 & 291 \\
\hline V1425 Aql & $15 \mathrm{~A}-27 \mathrm{~A}$ & 2015-03-08 & 11:17:05 & 290 & 11:31:00 & 294 & $11: 24: 03$ & 291 \\
\hline HY Leo & $15 \mathrm{~A}-20 \mathrm{~B}$ & 2015-03-09 & $07: 18: 40$ & 290 & 07:05:36 & 288 & $07: 12: 06$ & 291 \\
\hline Leo 1 & $15 \mathrm{~A}-20 \mathrm{~B}$ & 2015-03-09 & $07: 24: 25$ & 290 & $07: 37: 33$ & 285 & $07: 30: 57$ & 294 \\
\hline V1007 Her & $15 \mathrm{~A}-25 \mathrm{~A}$ & 2015-03-09 & $08: 29: 35$ & 290 & $08: 16: 36$ & 291 & 08:23:06 & 291 \\
\hline V1323 Her & $15 \mathrm{~A}-25 \mathrm{~A}$ & 2015-03-09 & 08:36:05 & 290 & 08:49:00 & 294 & & \\
\hline Mon 2 & $15 \mathrm{~A}-11 \mathrm{~A}$ & 2015-03-10 & & & $07: 18: 57$ & 291 & $07: 25: 36$ & 291 \\
\hline WX Pyx & $15 \mathrm{~A}-11 \mathrm{~A}$ & 2015-03-10 & & & $07: 52: 51$ & 291 & $07: 46: 21$ & 291 \\
\hline
\end{tabular}


Table 1 (continued)

\begin{tabular}{|c|c|c|c|c|c|c|c|c|}
\hline \multirow[t]{2}{*}{ GVCS Name } & \multirow[t]{2}{*}{ Semester } & \multirow[b]{2}{*}{$\begin{array}{c}\text { Date } \\
\text { UT }\end{array}$} & \multicolumn{2}{|c|}{ C-Band } & \multicolumn{2}{|c|}{ X-Band } & \multicolumn{2}{|c|}{ K-Band } \\
\hline & & & $\begin{array}{c}\text { Start } \\
\text { UT }\end{array}$ & $\begin{array}{c}\text { Expo } \\
(\mathrm{s})\end{array}$ & $\begin{array}{c}\text { Start } \\
\text { UT }\end{array}$ & $\begin{array}{c}\text { Expo } \\
(\mathrm{s})\end{array}$ & $\begin{array}{c}\text { Start } \\
\text { UT }\end{array}$ & $\begin{array}{c}\text { Expo } \\
(\mathrm{s})\end{array}$ \\
\hline VZ Sex & $15 \mathrm{~A}-19 \mathrm{~A}$ & 2015-03-10 & 09:53:00 & 280 & 09:40:09 & 282 & $09: 46: 33$ & 282 \\
\hline YY Sex & 15A-19A & 2015-03-10 & 10:00:20 & 280 & 10:13:30 & 279 & 10:06:54 & 282 \\
\hline V1007 Her & $15 \mathrm{~A}-25 \mathrm{~B}$ & 2015-03-10 & $08: 33: 15$ & 290 & $08: 20: 18$ & 291 & $08: 26: 45$ & 291 \\
\hline V1323 Her & $15 \mathrm{~A}-25 \mathrm{~B}$ & 2015-03-10 & 08:39:45 & 290 & $08: 52: 42$ & 291 & $08: 46: 12$ & 291 \\
\hline V4738 Sgr & $15 \mathrm{~A}-35 \mathrm{~B}$ & 2015-03-15 & $15: 52: 55$ & 115 & $15: 45: 48$ & 111 & $15: 49: 18$ & 117 \\
\hline Vir 1 & $15 \mathrm{~A}-22 \mathrm{~A}$ & 2015-03-28 & $08: 43: 35$ & 310 & $08: 29: 36$ & 309 & $08: 36: 33$ & 312 \\
\hline Vir 2 & $15 \mathrm{~A}-22 \mathrm{~A}$ & 2015-03-28 & $08: 49: 45$ & 305 & 09:03:39 & 312 & $08: 56: 42$ & 309 \\
\hline LW Cam & $15 \mathrm{~A}-10 \mathrm{~B}$ & 2015-03-31 & $07: 54: 40$ & 305 & $07: 41: 18$ & 300 & $07: 47: 57$ & 306 \\
\hline HT Cam & $15 \mathrm{~A}-10 \mathrm{~B}$ & 2015-03-31 & 08:01:55 & 305 & $08: 15: 51$ & 303 & 08:08:54 & 306 \\
\hline V1500 Cyg & $15 \mathrm{~A}-31 \mathrm{~A}$ & 2015-04-06 & $14: 28: 05$ & 250 & $14: 15: 24$ & 246 & $14: 21: 42$ & 252 \\
\hline V2069 Cyg & $15 \mathrm{~A}-31 \mathrm{~A}$ & 2015-04-06 & $14: 35: 50$ & 250 & $14: 47: 57$ & 246 & $14: 41: 54$ & 252 \\
\hline AE Aqr & $15 \mathrm{~A}-30 \mathrm{~A}$ & 2015-04-09 & $13: 54: 25$ & 285 & $13: 41: 03$ & 294 & $13: 47: 42$ & 291 \\
\hline Aqr 1 & $15 \mathrm{~A}-30 \mathrm{~A}$ & 2015-04-09 & 14:00:10 & 285 & 14:13:09 & 285 & 14:06:39 & 288 \\
\hline LS Cam & 15A-08B & 2015-04-10 & $07: 46: 20$ & 305 & $07: 32: 48$ & 303 & $07: 39: 33$ & 306 \\
\hline HS Cam & $15 \mathrm{~A}-08 \mathrm{~B}$ & 2015-04-10 & $07: 53: 10$ & 305 & 08:06:48 & 300 & 08:00:00 & 306 \\
\hline Tau 4 & $15 \mathrm{~A}-05 \mathrm{~A}$ & 2015-04-12 & $18: 57: 00$ & 310 & $19: 10: 54$ & 318 & 19:03:57 & 315 \\
\hline V1062 Tau & $15 \mathrm{~A}-05 \mathrm{~A}$ & 2015-04-12 & $18: 50: 35$ & 315 & $18: 36: 06$ & 315 & $18: 43: 18$ & 318 \\
\hline UU Col & $15 \mathrm{~A}-06 \mathrm{~A}$ & 2015-04-12 & $23: 49: 00$ & 300 & $23: 35: 06$ & 303 & $23: 42: 00$ & 306 \\
\hline Lep 1 & $15 \mathrm{~A}-06 \mathrm{~A}$ & 2015-04-12 & $23: 55: 05$ & 300 & 00:08:36 & 303 & 00:01:48 & 306 \\
\hline V388 Peg & $15 \mathrm{~A}-33 \mathrm{~A}$ & 2015-04-16 & $15: 26: 10$ & 285 & $15: 13: 21$ & 288 & $15: 19: 45$ & 288 \\
\hline Aqr 2 & $15 \mathrm{~A}-33 \mathrm{~A}$ & 2015-04-16 & 15:33:05 & 285 & $15: 46: 12$ & 285 & 15:39:39 & 285 \\
\hline MT Dra & $15 \mathrm{~A}-28 \mathrm{~A}$ & 2015-04-17 & 09:04:15 & 280 & 08:51:00 & 279 & $08: 57: 36$ & 282 \\
\hline EP Dra & $15 \mathrm{~A}-28 \mathrm{~A}$ & 2015-04-17 & 09:11:20 & 280 & 09:24:09 & 279 & $09: 17: 45$ & 279 \\
\hline V2731 Oph & $15 \mathrm{~A}-26 \mathrm{~A}$ & 2015-04-21 & $12: 24: 00$ & 280 & 12:11:09 & 282 & $12: 17: 33$ & 282 \\
\hline Sgr 1 & $15 \mathrm{~A}-26 \mathrm{~A}$ & 2015-04-21 & $12: 31: 25$ & 275 & 12:44:00 & 282 & $12: 37: 42$ & 279 \\
\hline EV Lyn & $15 \mathrm{~A}-12 \mathrm{~A}$ & 2015-04-24 & $06: 31: 55$ & 310 & $06: 17: 54$ & 315 & $06: 24: 54$ & 315 \\
\hline EG Lyn & $15 \mathrm{~A}-12 \mathrm{~A}$ & 2015-04-24 & 06:38:00 & 315 & $06: 51: 57$ & 318 & 06:45:00 & 315 \\
\hline V388 Peg & $15 \mathrm{~A}-33 \mathrm{~B}$ & $2015-04-25$ & $14: 16: 55$ & 285 & 14:04:06 & 285 & $14: 10: 30$ & 285 \\
\hline Aqr 2 & 15A-33B & 2015-04-25 & $14: 23: 50$ & 285 & $14: 36: 54$ & 288 & $14: 30: 21$ & 288 \\
\hline V2731 Oph & $15 \mathrm{~A}-26 \mathrm{~B}$ & 2015-04-28 & $08: 20: 55$ & 280 & 08:08:09 & 282 & $08: 14: 33$ & 282 \\
\hline Sgr 1 & $15 \mathrm{~A}-26 \mathrm{~B}$ & 2015-04-28 & $08: 28: 20$ & 280 & $08: 40: 57$ & 282 & 08:34:39 & 282 \\
\hline V407 Vul & $15 \mathrm{~A}-29 \mathrm{~A}$ & 2015-04-28 & $14: 31: 05$ & 270 & $14: 18: 39$ & 261 & $14: 24: 48$ & 270 \\
\hline V2306 Cyg & $15 \mathrm{~A}-29 \mathrm{~A}$ & 2015-04-28 & 14:38:05 & 270 & $14: 50: 42$ & 261 & $14: 44: 24$ & 270 \\
\hline AE Aqr & $15 \mathrm{~A}-30 \mathrm{~B}$ & 2015-04-28 & $13: 31: 45$ & 290 & $13: 18: 27$ & 291 & $13: 25: 06$ & 291 \\
\hline Aqr 1 & 15A-30B & 2015-04-28 & $13: 37: 35$ & 285 & $13: 50: 33$ & 285 & $13: 44: 03$ & 288 \\
\hline EV Lyn & $15 \mathrm{~A}-12 \mathrm{~B}$ & 2015-04-29 & $06: 16: 40$ & 315 & 06:02:39 & 318 & 06:09:39 & 318 \\
\hline EG Lyn & $15 \mathrm{~A}-12 \mathrm{~B}$ & 2015-04-29 & $06: 22: 50$ & 310 & $06: 36: 45$ & 315 & $06: 29: 45$ & 318 \\
\hline Vir 1 & $15 \mathrm{~A}-22 \mathrm{~B}$ & 2015-05-01 & $06: 29: 50$ & 310 & $06: 15: 51$ & 312 & $06: 22: 51$ & 312 \\
\hline Vir 2 & $15 \mathrm{~A}-22 \mathrm{~B}$ & 2015-05-01 & 06:36:00 & 310 & $06: 49: 57$ & 312 & $06: 42: 57$ & 312 \\
\hline V1500 Cyg & 15A-31B & 2015-05-01 & $12: 51: 35$ & 250 & $12: 38: 54$ & 246 & $12: 45: 12$ & 252 \\
\hline V2069 Cyg & $15 \mathrm{~A}-31 \mathrm{~B}$ & 2015-05-01 & $12: 59: 20$ & 250 & $13: 11: 27$ & 246 & $13: 05: 24$ & 252 \\
\hline
\end{tabular}


Table 1 (continued)

\begin{tabular}{|c|c|c|c|c|c|c|c|c|}
\hline \multirow[t]{2}{*}{ GVCS Name } & \multirow[t]{2}{*}{ Semester } & \multirow[b]{2}{*}{$\begin{array}{c}\text { Date } \\
\text { UT }\end{array}$} & \multicolumn{2}{|c|}{ C-Band } & \multicolumn{2}{|c|}{ X-Band } & \multicolumn{2}{|c|}{ K-Band } \\
\hline & & & $\begin{array}{c}\text { Start } \\
\text { UT }\end{array}$ & $\begin{array}{c}\text { Expo } \\
\text { (s) }\end{array}$ & $\begin{array}{c}\text { Start } \\
\text { UT }\end{array}$ & $\begin{array}{c}\text { Expo } \\
\text { (s) }\end{array}$ & $\begin{array}{c}\text { Start } \\
\text { UT }\end{array}$ & $\begin{array}{c}\text { Expo } \\
\text { (s) }\end{array}$ \\
\hline VZ Sex & 15A-19B & 2015-05-02 & $06: 24: 35$ & 280 & $06: 1$ & 282 & 06:18:09 & 282 \\
\hline YY Sex & 15A-19B & 2015-05-02 & 06:32:00 & 280 & $06: 45: 06$ & 282 & $06: 38: 33$ & 279 \\
\hline IQ Eri & $15 \mathrm{~A}-03 \mathrm{~A}$ & 2015-05-04 & $16: 17: 50$ & 290 & 16:04:45 & 285 & $16: 11: 12$ & 294 \\
\hline UZ For & $15 \mathrm{~A}-03 \mathrm{~A}$ & 2015-05-04 & $16: 24: 40$ & 290 & $16: 37: 48$ & 288 & $16: 31: 15$ & 291 \\
\hline $\mathrm{AP} \mathrm{CrB}$ & $15 \mathrm{~A}-23 \mathrm{~A}$ & 2015-05-06 & $11: 56: 50$ & 275 & 11:44:06 & 276 & $11: 50: 24$ & 282 \\
\hline V1189 Her & $15 \mathrm{~A}-23 \mathrm{~A}$ & 2015-05-06 & $12: 03: 10$ & 275 & $12: 17: 12$ & 276 & 12:10:06 & 282 \\
\hline AP CrB & $15 \mathrm{~A}-23 \mathrm{~B}$ & 2015-05-07 & $12: 25: 50$ & 275 & 12:13:06 & 276 & $12: 19: 24$ & 282 \\
\hline V1189 Her & $15 \mathrm{~A}-23 \mathrm{~B}$ & 2015-05-07 & $12: 32: 10$ & 275 & $12: 46: 12$ & 276 & 12:39:06 & 282 \\
\hline Her 1 & $15 \mathrm{~A}-24 \mathrm{~A}$ & 2015-05-07 & $04: 20: 30$ & 300 & 04:07:15 & 297 & 04:13:48 & 303 \\
\hline V1237 Her & $15 \mathrm{~A}-24 \mathrm{~A}$ & 2015-05-07 & $04: 27: 25$ & 295 & 04:40:48 & 297 & 04:34:06 & 300 \\
\hline Her 1 & $15 \mathrm{~A}-24 \mathrm{~B}$ & 2015-05-08 & 04:11:25 & 295 & 03:58:09 & 297 & 04:04:42 & 303 \\
\hline V1237 Her & $15 \mathrm{~A}-24 \mathrm{~B}$ & 2015-05-08 & $04: 18: 15$ & 300 & 04:31:42 & 297 & $04: 25: 00$ & 300 \\
\hline FH Uma & $15 \mathrm{~A}-21 \mathrm{~B}$ & 2015-05-10 & $06: 22: 20$ & 265 & 06:10:00 & 267 & 06:16:09 & 267 \\
\hline EK Uma & & 2015-05-10 & $06: 28: 50$ & 265 & 06:41:06 & 267 & $06: 34: 57$ & 267 \\
\hline V407 Vul & 15A-29B & 2015-05-10 & $15: 12: 25$ & 265 & $14: 59: 54$ & 261 & 15:06:03 & 270 \\
\hline V2306 C & & 2015-05-10 & $15: 19: 20$ & 270 & $15: 32: 00$ & 261 & $15: 25: 39$ & 273 \\
\hline MT Dra & $15 \mathrm{~A}-28 \mathrm{~B}$ & 2015-05-11 & $11: 34: 30$ & 280 & $11: 21: 12$ & 282 & $11: 27: 51$ & 282 \\
\hline EP Dra & & 2015-05-11 & $11: 41: 35$ & 280 & $11: 54: 24$ & 279 & 11:48:00 & 279 \\
\hline Sct 1 & 15A-27B & 2015-05-16 & $09: 24: 20$ & 290 & 09:11:21 & 291 & $09: 17: 51$ & 291 \\
\hline V1425 & & 2015-05-16 & & 290 & & 291 & & 91 \\
\hline Aqr 5 & $15 \mathrm{~A}-34 \mathrm{~A}$ & 2015-05-24 & $14: 26: 50$ & 265 & $14: 14: 39$ & 267 & $14: 20: 45$ & 264 \\
\hline BW Scl & $15 \mathrm{~A}-34 \mathrm{~A}$ & 2015-05-24 & $14: 34: 35$ & 295 & $14: 47: 48$ & 294 & 14:41:09 & 03 \\
\hline EQ Cet & $15 \mathrm{~A}-01 \mathrm{~A}$ & 2015-05-25 & $14: 29: 55$ & 290 & $14: 16: 42$ & 285 & $14: 23: 15$ & 291 \\
\hline VW For & $15 \mathrm{~A}-01 \mathrm{~A}$ & 2015-05-25 & $14: 36: 55$ & 290 & $14: 50: 21$ & 285 & $14: 43: 36$ & 294 \\
\hline Aqr 5 & $15 \mathrm{~A}-34 \mathrm{~B}$ & 2015-05-25 & $13: 29: 40$ & 265 & $13: 17: 33$ & 264 & $13: 23: 36$ & 267 \\
\hline BW Sc & $15 \mathrm{~A}-34 \mathrm{~B}$ & 2015-05-25 & $13: 37: 25$ & 300 & 13:50:42 & 291 & 13:44:03 & 300 \\
\hline Tau 4 & $15 \mathrm{~A}-05 \mathrm{~B}$ & 2015-05-31 & $14: 52: 15$ & 315 & $15: 06: 12$ & 318 & $14: 59: 15$ & 315 \\
\hline V1062 Tau & $15 \mathrm{~A}-05 \mathrm{~B}$ & 2015-05-31 & $14: 45: 55$ & 310 & $14: 31: 24$ & 315 & $14: 38: 39$ & 315 \\
\hline Aur (Paloma) & $15 \mathrm{~A}-07 \mathrm{~A}$ & 2015-06-01 & $15: 05: 30$ & 280 & $14: 51: 45$ & 285 & $14: 58: 33$ & 291 \\
\hline V647 Aur & $15 \mathrm{~A}-07 \mathrm{~A}$ & 2015-06-01 & $15: 12: 35$ & 285 & $15: 25: 39$ & 285 & 15:19:06 & 291 \\
\hline Aur (Paloma) & 15A-07B & 2015-06-01 & $17: 05: 10$ & 285 & $16: 51: 27$ & 285 & $16: 58: 15$ & 291 \\
\hline V647 Aur & 15A-07B & 2015-06-01 & $17: 12: 20$ & 285 & $17: 25: 21$ & 288 & $17: 18: 48$ & 291 \\
\hline V418 Gem & 15A-09A & 2015-06-01 & $16: 14: 15$ & 285 & $16: 00: 33$ & 291 & $16: 07: 24$ & 291 \\
\hline EU Lyn & 15A-09A & 2015-06-01 & $16: 20: 10$ & 290 & $16: 33: 48$ & 291 & $16: 27: 00$ & 291 \\
\hline Mon 2 & 15A-11B & 2015-06-03 & $00: 38: 40$ & 290 & $00: 25: 24$ & 291 & $00: 32: 03$ & 291 \\
\hline WX Pyx & $15 \mathrm{~A}-11 \mathrm{~B}$ & 2015-06-03 & $00: 46: 20$ & 290 & $00: 59: 18$ & 291 & $00: 52: 48$ & 291 \\
\hline EQ Cet & 15A-01B & 2015-06-12 & $13: 31: 40$ & 290 & $13: 18: 27$ & 288 & $13: 25: 00$ & 294 \\
\hline VW For & 15A-01B & 2015-06-12 & $13: 38: 40$ & 290 & $13: 52: 06$ & 288 & $13: 45: 24$ & 291 \\
\hline V418 Gem & 15A-09B & 2015-06-14 & $15: 16: 20$ & 290 & $15: 02: 42$ & 291 & 15:09:33 & 291 \\
\hline EU Lyn & 15A-09B & 2015-06-14 & $15: 22: 20$ & 290 & $15: 35: 57$ & 291 & 15:29:09 & 291 \\
\hline EU Cnc & $15 \mathrm{~A}-15 \mathrm{~A}$ & 2015-06-14 & $17: 30: 35$ & 295 & $17: 17: 03$ & 291 & $17: 23: 48$ & 294 \\
\hline
\end{tabular}


JVLA MCV SuRvey Data

Table 1 (continued)

\begin{tabular}{|c|c|c|c|c|c|c|c|c|}
\hline \multirow[t]{2}{*}{ GVCS Name } & \multirow[t]{2}{*}{ Semester } & \multirow[b]{2}{*}{$\begin{array}{c}\text { Date } \\
\text { UT }\end{array}$} & \multicolumn{2}{|c|}{ C-Band } & \multicolumn{2}{|c|}{ X-Band } & \multicolumn{2}{|c|}{ K-Band } \\
\hline & & & $\begin{array}{c}\text { Start } \\
\text { UT }\end{array}$ & $\begin{array}{c}\text { Expo } \\
\text { (s) }\end{array}$ & $\begin{array}{c}\text { Start } \\
\text { UT }\end{array}$ & $\begin{array}{c}\text { Expo } \\
\text { (s) }\end{array}$ & $\begin{array}{c}\text { Start } \\
\text { UT }\end{array}$ & $\begin{array}{c}\text { Expo } \\
\text { (s) }\end{array}$ \\
\hline HS $0922+1333$ & $15 \mathrm{~A}-15 \mathrm{~A}$ & 2015-06-14 & $17: 36: 35$ & 295 & $17: 50: 33$ & 291 & $17: 43: 33$ & 297 \\
\hline MN Hya & $15 \mathrm{~A}-17 \mathrm{~A}$ & 2015-06-14 & 20:12:05 & 280 & 19:59:15 & 282 & 20:05:39 & 282 \\
\hline Hya 3 & $15 \mathrm{~A}-17 \mathrm{~A}$ & 2015-06-14 & $20: 18: 50$ & 280 & $20: 31: 39$ & 282 & $20: 25: 15$ & 282 \\
\hline IQ Eri & 15A-03B & 2015-06-15 & $13: 17: 10$ & 290 & 13:04:06 & 285 & $13: 10: 36$ & 291 \\
\hline UZ For & $15 \mathrm{~A}-03 \mathrm{~B}$ & 2015-06-15 & $13: 24: 05$ & 290 & $13: 37: 12$ & 285 & $13: 30: 36$ & 291 \\
\hline Lyn 1 & $15 \mathrm{~A}-14 \mathrm{~B}$ & 2015-06-28 & $17: 33: 25$ & 280 & $17: 20: 21$ & 282 & $17: 26: 51$ & 285 \\
\hline Cnc 2 & $15 \mathrm{~A}-14 \mathrm{~B}$ & 2015-06-28 & $17: 40: 35$ & 280 & $17: 53: 27$ & 282 & $17: 46: 57$ & 288 \\
\hline DW Cnc & $15 \mathrm{~A}-13 \mathrm{~A}$ & 2015-07-02 & $15: 39: 25$ & 300 & $15: 53: 03$ & 300 & $15: 46: 12$ & 303 \\
\hline Cnc 1 & $15 \mathrm{~A}-13 \mathrm{~A}$ & 2015-07-02 & $15: 33: 25$ & 300 & $15: 19: 48$ & 300 & $15: 26: 36$ & 303 \\
\hline And 1 & $15 \mathrm{~A}-32 \mathrm{~A}$ & 2015-07-02 & $16: 32: 10$ & 285 & $16: 44: 54$ & 276 & $16: 38: 30$ & 291 \\
\hline Cyg 1 & $15 \mathrm{~A}-32 \mathrm{~A}$ & 2015-07-02 & $16: 23: 45$ & 290 & 16:09:30 & 276 & $16: 16: 30$ & 291 \\
\hline UU Col & & 2015-07-03 & $15: 34: 35$ & 300 & $15: 20: 45$ & 300 & $15: 27: 39$ & 306 \\
\hline Lep 1 & $15 \mathrm{~A}-06 \mathrm{~B}$ & 2015-07-03 & $15: 40: 40$ & 300 & $15: 54: 12$ & 303 & $15: 47: 24$ & 306 \\
\hline And 1 & $15 \mathrm{~A}-32 \mathrm{~B}$ & 2015-07-21 & $14: 02: 40$ & 285 & $14: 15: 24$ & 276 & 14:09:00 & 291 \\
\hline Cyg 1 & $15 \mathrm{~A}-32 \mathrm{~B}$ & 2015-07-21 & $13: 54: 15$ & 290 & 13:40:00 & 276 & $13: 47: 00$ & 291 \\
\hline HY Eri & $15 \mathrm{~A}-04 \mathrm{~A}$ & $2015-07-25$ & $14: 22: 25$ & 280 & 14:09:30 & 282 & $14: 15: 54$ & 285 \\
\hline Mon 1 & $15 \mathrm{~A}-04 \mathrm{~A}$ & 2015-07-25 & 14:30:05 & 275 & $14: 43: 24$ & 282 & $14: 36: 42$ & 285 \\
\hline DW Cn & 15A-13B & $2015-07-25$ & 13:39:05 & 300 & $13: 52: 42$ & 300 & $13: 45: 54$ & 300 \\
\hline Cnc 1 & $15 \mathrm{~A}-13 \mathrm{~B}$ & $2015-07-25$ & 13:33:05 & 300 & $13: 19: 27$ & 303 & $13: 26: 15$ & 303 \\
\hline HY Eri & 15A-04B & 2015-07-26 & $13: 31: 20$ & 280 & $13: 18: 24$ & 282 & $13: 24: 48$ & 288 \\
\hline Mon 1 & $15 \mathrm{~A}-04 \mathrm{~B}$ & $2015-07-26$ & 13:39:00 & 275 & $13: 52: 18$ & 282 & $13: 45: 36$ & 285 \\
\hline $\mathrm{EU}$ Cnc & 15A-15B & $2015-07-28$ & $14: 50: 10$ & 295 & $14: 36: 36$ & 291 & $14: 43: 18$ & 297 \\
\hline HS $0922+1333$ & $15 \mathrm{~A}-15 \mathrm{~B}$ & $2015-07-28$ & $14: 56: 10$ & 290 & $15: 10: 06$ & 291 & 15:03:06 & 297 \\
\hline BS Tri & $15 \mathrm{~A}-02 \mathrm{~B}$ & 2015-08-03 & $14: 48: 10$ & 290 & $14: 34: 42$ & 291 & $14: 41: 27$ & 291 \\
\hline XY Ari & $15 \mathrm{~A}-02 \mathrm{~B}$ & 2015-08-03 & $14: 55: 25$ & 290 & $15: 08: 33$ & 291 & $15: 01: 57$ & 291 \\
\hline GZ Cnc & $15 \mathrm{~A}-16 \mathrm{~A}$ & 2015-08-06 & $14: 43: 15$ & 290 & $14: 29: 51$ & 288 & $14: 36: 30$ & 291 \\
\hline HU Leo & $15 \mathrm{~A}-16 \mathrm{~A}$ & 2015-08-06 & $14: 49: 10$ & 290 & $15: 02: 36$ & 288 & $14: 55: 54$ & 291 \\
\hline MN Hya & $15 \mathrm{~A}-17 \mathrm{~B}$ & 2015-08-21 & $15: 49: 00$ & 280 & $15: 36: 12$ & 282 & $15: 42: 36$ & 282 \\
\hline Hya 3 & 15A-17B & 2015-08-21 & $15: 55: 50$ & 280 & $16: 08: 36$ & 282 & $16: 02: 12$ & 282 \\
\hline GZ Cnc & $15 \mathrm{~A}-16 \mathrm{~B}$ & 2015-09-23 & 11:53:00 & 290 & 11:39:39 & 285 & $11: 46: 18$ & 291 \\
\hline HU Leo & 15A-16B & 2015-09-23 & $11: 58: 55$ & 290 & $12: 12: 24$ & 285 & 12:05:39 & 291 \\
\hline US 691 & $15 \mathrm{~A}-18 \mathrm{~A}$ & 2015-09-27 & $15: 10: 45$ & 255 & $14: 58: 39$ & 258 & 15:04:39 & 261 \\
\hline WX Lmi & $15 \mathrm{~A}-18 \mathrm{~A}$ & 2015-09-27 & $15: 18: 25$ & 250 & $15: 30: 24$ & 258 & $15: 24: 21$ & 261 \\
\hline US 691 & $15 \mathrm{~A}-18 \mathrm{~B}$ & 2015-10-05 & $14: 34: 40$ & 255 & $14: 22: 36$ & 255 & $14: 28: 33$ & 261 \\
\hline WX Lmi & 15A-18B & 2015-10-05 & $14: 42: 20$ & 250 & $14: 54: 21$ & 255 & $14: 48: 15$ & 261 \\
\hline
\end{tabular}

All data are calibrated using CASA version 4.2.2 and the JVLA calibration pipeline version 1.3.1. Although scheduling blocks $15-17$ are not properly flux calibrated because CASA does not have models for the flux standard, 3C295; the flux density estimates of these data do not appear to be grossly different compared to the properly calibrated data. Note that an incorrect flux calibration does not affect the presence of a radio source. The resulting calibrated images were then cleaned using an automated script. An image size of $2500 \times 2500$ pixels 
was used at a scale of 0.02 arcseconds per pixel, resulting in a $50 \times 50$ arcsecond image. Six sets of uncleaned images were created; specifically, I, Q, U, V, LL, and RR. Natural weighting was used for all images. For a two minute integration, RMS errors of 25,25 , and 33 $\mu \mathrm{Jy} /$ beam are expected for the $\mathrm{C}-, \mathrm{X}-$, and K-bands, respectively.

\section{RESULTS}

Table 2 lists the 121 MCVs observed during this survey and their CV subclass and optical positions. As in Table 1 , column 1 is the GCVS name. Column 2 is the CV subclass. Columns 3 and 4 are the right ascension (RA) and declination (Dec) of the star at epoch J2000. Columns 5 and 6 are the proper motion of the star, if known. Proper motions are primarily from the US Naval Observatory Combined Astrometric Catalog (UCAC4) supplemented by the Simbad on-line catalog when necessary. Columns 7 and 8 are the RA and Dec of the star at the epoch of the observation. The last two columns enable a comparison of the observed position to the cataloged position.

Table 2. Target list and their properties

\begin{tabular}{|c|c|c|c|c|c|c|c|}
\hline GCVS Name & Class $^{\mathrm{a}}$ & $\begin{array}{c}\text { RA } \\
(\text { hh mm ss.ss) }\end{array}$ & $\begin{array}{c}\text { Dec } \\
(\mathrm{dd} \text { mm ss.s })\end{array}$ & $\begin{array}{c}\text { PM RA } \\
\text { (mas) }\end{array}$ & $\begin{array}{c}\text { PM Dec } \\
\text { (mas) }\end{array}$ & $\begin{array}{c}\mathrm{RA}+\mathrm{PM} \\
(\mathrm{hh} \mathrm{mm} \text { ss.ss})\end{array}$ & $\begin{array}{c}\text { Dec+PM } \\
(\mathrm{dd} \text { mm ss.s })\end{array}$ \\
\hline V709 Cas & IP & 002848.84 & +591722.3 & 0.2 & -1.8 & 002848.84 & +591722.3 \\
\hline And 1 & IP ? & 004308.61 & +411247.6 & 7.1 & 1.5 & 004308.62 & +411247.6 \\
\hline EQ Cet & $\mathrm{AM}$ & 012852.52 & -233943.8 & 29 & -34 & 012852.55 & -233944.3 \\
\hline Cas 1 & IP & 015320.76 & +744622.2 & & & 015320.76 & +744622.2 \\
\hline FL Cet & $\mathrm{AM}$ & 015543.44 & +002806.4 & 64.3 & 13.9 & 015543.50 & +002806.6 \\
\hline AI Tri & $\mathrm{AM}$ & 020348.60 & +295926.0 & -8.5 & -16.2 & 020348.59 & +295925.8 \\
\hline TT Ari & VY & 020653.09 & +151741.8 & -8 & -24.2 & 020653.08 & +151741.5 \\
\hline BS Tri & $\mathrm{AM}$ & 020929.80 & +283229.3 & & & 020929.80 & +283229.3 \\
\hline VW For & $\mathrm{AM} ?$ & 025251.35 & -303742.6 & 8.6 & -30.1 & 025251.36 & $\begin{array}{lll}-30 & 37 & 43.1\end{array}$ \\
\hline IQ Eri & $\mathrm{DN}$ & 025538.03 & -224702.7 & 29 & -1.9 & 025538.06 & $\begin{array}{lll}-22 & 47 & 02.7\end{array}$ \\
\hline XY Ari & IP & 025608.19 & +192634.1 & & & 025608.19 & +192634.1 \\
\hline EF Eri & $\mathrm{AM}$ & 031413.26 & -223543.3 & 123 & -47 & 031413.38 & -223544.0 \\
\hline GK Per & IP & 033112.01 & +435415.4 & -8 & -17.9 & 033112.00 & +435415.2 \\
\hline VY For & $\mathrm{AM}$ & 033204.61 & -255655.0 & & & 033204.61 & -255655.0 \\
\hline UZ For & $\mathrm{AM}$ & 033528.62 & -254422.3 & & & 033528.62 & -254422.3 \\
\hline V1294 Tau & VY & 040037.23 & +062246.3 & 2.9 & -4.4 & 040037.23 & +062246.2 \\
\hline AH Eri & IP ? & 042238.05 & -132130.5 & & & 042238.05 & -132130.5 \\
\hline IW Eri & $\mathrm{AM}$ & 042555.18 & -194530.4 & & & 042555.18 & -194530.4 \\
\hline HY Eri & $\mathrm{AM}$ & 050146.39 & -035920.7 & & & 050146.39 & -03 5920.7 \\
\hline V1062 Tau & IP & $0502 \quad 27.47$ & +244523.4 & -4.1 & 0.8 & $0502 \quad 27.47$ & +244523.4 \\
\hline Tau 4 & $\mathrm{AM} ?$ & 050250.94 & +162422.0 & 2.9 & -5 & 050250.94 & +162421.9 \\
\hline UU Col & IP & 051213.22 & -324139.8 & -2.2 & 11 & $\begin{array}{lll}05 & 12 & 13.22\end{array}$ & -324139.6 \\
\hline V1309 Ori & $\mathrm{AM}$ & $05 \quad 1541.41$ & +010440.4 & 4.1 & -8.2 & $05 \quad 1541.41$ & +010440.3 \\
\hline Aur (Paloma) & $\mathrm{AM}$ & 052430.48 & +424450.2 & 4.2 & 7 & 052430.48 & +424450.3 \\
\hline TV Col & IP & 052925.52 & -324904.0 & 13.3 & 16.9 & 052925.53 & $\begin{array}{lll}-32 & 49 & 03.8\end{array}$ \\
\hline BY Cam & $\mathrm{AM}$ & 054248.77 & +605131.5 & -41 & 10.5 & 054248.73 & +605131.6 \\
\hline LS Cam & IP ? & 055723.93 & +724152.6 & -3.8 & 9.4 & 055723.93 & +724152.7 \\
\hline V405 Aur & IP & 055759.27 & +535345.1 & -2.6 & -12.9 & 055759.27 & +535344.9 \\
\hline Lep 1 & $\mathrm{AM} ?$ & 060033.30 & -270918.5 & -5 & 0.1 & 060033.29 & -270918.5 \\
\hline V552 Aur & IP ? & 061409.81 & +453008.5 & 7.7 & -7.3 & 061409.82 & +453008.4 \\
\hline MU Cam & IP & 062516.18 & +733439.2 & 3.5 & 4.5 & 062516.18 & +733439.3 \\
\hline
\end{tabular}


JVLA MCV Survey Data

Table 2 (continued)

\begin{tabular}{|c|c|c|c|c|c|c|c|}
\hline GCVS Name & Class $^{\mathrm{a}}$ & $\begin{array}{c}\text { RA } \\
\text { (hh mm ss.ss) }\end{array}$ & $\begin{array}{c}\text { Dec } \\
\text { (dd mm ss.s) }\end{array}$ & $\begin{array}{l}\text { PM RA } \\
\text { (mas) }\end{array}$ & $\begin{array}{l}\text { PM Dec } \\
\text { (mas) }\end{array}$ & $\begin{array}{c}\mathrm{RA}+\mathrm{PM} \\
(\mathrm{hh} \mathrm{mm} \text { ss.ss) }\end{array}$ & $\begin{array}{c}\text { Dec+PM } \\
\text { (dd mm ss.s) }\end{array}$ \\
\hline V647 Aur & IP & 063632.55 & +353543.3 & 1.5 & 0.9 & 063632.55 & +353543.3 \\
\hline Mon 1 & $\mathrm{AM}$ & 064950.88 & -073741.7 & 6.7 & 8.7 & 064950.89 & -073741.6 \\
\hline V418 Gem & IP & 070408.67 & +262510.9 & 1.9 & 1.8 & 070408.67 & +262510.9 \\
\hline LW Cam & $\mathrm{AM}$ & 070409.98 & +620328.3 & 9.7 & -12.9 & 070409.99 & +620328.1 \\
\hline V348 Pup & SW & 071232.90 & -360538.6 & 1.4 & 8.1 & 071232.90 & -360538.5 \\
\hline HS Cam & $\mathrm{AM}$ & 071914.49 & +655745.0 & & & 071914.49 & +655745.0 \\
\hline GI Mon & IP ? & 072647.10 & -064029.5 & 7 & 5.4 & 072647.11 & -06 4029.4 \\
\hline BG CMi & $\mathrm{AM}$ & 073129.00 & +09 5623.1 & -15 & -21.1 & 073128.99 & +095622.8 \\
\hline Mon 2 & $\mathrm{AM}$ & 074910.40 & -054925.6 & -1.5 & 3.3 & 074910.40 & -054925.5 \\
\hline PQ Gem & IP & 075117.33 & +144423.9 & -4.3 & 7.7 & $0751 \quad 17.33$ & +144424.0 \\
\hline EU Lyn & $\mathrm{AM}$ & 075240.45 & +362823.2 & -0.4 & 5.5 & 075240.45 & +362823.3 \\
\hline EV Lyn & SW ? & 075443.01 & +5007 29.2 & -3 & -5.9 & 075443.01 & +500729.1 \\
\hline HT Cam & IP & 075701.37 & +630601.8 & -3.4 & -1.1 & 075701.37 & +630601.8 \\
\hline DW Cnc & IP & 075853.03 & +161645.2 & -20.1 & -3.4 & 075853.01 & +161645.1 \\
\hline Cnc 1 & $\mathrm{AM} ?$ & 075939.79 & +19 1417.3 & & & 075939.79 & +191417.3 \\
\hline V351 Pup & IP ? & 081138.40 & -350730.5 & & & 081138.40 & -350730.5 \\
\hline VV Pup & $\mathrm{AM}$ & 081506.78 & $\begin{array}{lll}-19 & 03 & 17.8\end{array}$ & 9.7 & -70.7 & 081506.79 & -190318.8 \\
\hline EG Lyn & $\mathrm{AM}$ & 082051.07 & +493431.7 & & & 082051.07 & +493431.7 \\
\hline WX Pyx & IP & 083305.74 & -224832.2 & -0.6 & 1.2 & 083305.74 & -224832.2 \\
\hline SW UMa & $\mathrm{SU}$ & 083642.74 & +532838.1 & & & 083642.74 & +532838.1 \\
\hline Lyn 1 & $\mathrm{AM}$ & 083751.00 & +383012.5 & -0.6 & 7.2 & 083751.00 & +383012.6 \\
\hline Cnc 2 & IP ? & 084617.12 & +243444.1 & & & 084617.12 & +243444.1 \\
\hline EU Cnc & $\mathrm{AM}$ & 085127.19 & +114657.0 & -13.8 & -6.9 & 085127.18 & +114656.9 \\
\hline Hya 1 & $\mathrm{AM}$ & 085909.18 & +053654.5 & & & 085909.18 & +053654.5 \\
\hline GZ Cnc & $\mathrm{SU}$ & 091551.67 & +090049.6 & -36.4 & -29.8 & 091551.63 & +090049.1 \\
\hline HU Leo & $\mathrm{AM} ?$ & 092444.48 & +08 0151.0 & 6.8 & -9.7 & 092444.49 & +080150.8 \\
\hline HS $0922+1333$ & $\mathrm{AM}$ & 092455.94 & +132052.4 & -17 & 2.6 & 092455.92 & +132052.4 \\
\hline MN Hya & $\mathrm{AM}$ & 092907.04 & -240505.4 & 8.3 & -0.9 & 092907.05 & -240505.4 \\
\hline US 691 & $\mathrm{SU}$ & 093249.57 & +472523.0 & & & 093249.57 & +472523.0 \\
\hline VZ Sex & IP ? & 094431.70 & +035805.6 & -18.3 & -17.9 & 094431.68 & +035805.3 \\
\hline HY Leo & IP & 094634.50 & +135058.1 & -9 & -1.5 & 094634.49 & +135058.1 \\
\hline Leo 1 & $\mathrm{AM}$ & 095308.17 & +145836.1 & & & 095308.17 & +145836.1 \\
\hline Hya 2 & $\mathrm{AM}$ & 100211.71 & -192537.4 & & & 100211.71 & -192537.4 \\
\hline Hya 3 & $\mathrm{AM}$ & 100734.64 & -201732.4 & & & 100734.64 & -201732.4 \\
\hline GG Leo & $\mathrm{AM}$ & 101534.67 & +090442.0 & -29.3 & -8.4 & 101534.64 & +0904 41.9 \\
\hline WX LMi & $\mathrm{AM}$ & 102627.49 & +384502.5 & 28 & -30 & 102627.52 & +384502.0 \\
\hline YY Sex & IP ? & 103946.97 & -050658.3 & -25.7 & -4 & 103946.96 & -050058.1 \\
\hline FH UMa & $\mathrm{AM}$ & 104709.93 & +633513.1 & & & 104709.93 & +633513.1 \\
\hline EK UMa & $\mathrm{AM}$ & 105135.15 & +540436.1 & & & 105135.15 & +540436.1 \\
\hline AN UMa & $\mathrm{AM}$ & 110425.67 & +450314.0 & -28.6 & -17.9 & 110425.64 & +450313.8 \\
\hline ST LMi & $\mathrm{AM}$ & 110539.76 & +250628.7 & -1.2 & -35.9 & 110539.76 & +250628.2 \\
\hline AR UMa & $\mathrm{AM}$ & 111544.56 & +425822.4 & -77 & 2 & 111544.49 & +425822.4 \\
\hline
\end{tabular}


Table 2 (continued)

\begin{tabular}{|c|c|c|c|c|c|c|c|}
\hline GCVS Name & Class $^{\mathrm{a}}$ & $\begin{array}{c}\text { RA } \\
\text { (hh mm ss.ss) }\end{array}$ & $\begin{array}{c}\text { Dec } \\
(\mathrm{dd} \text { mm ss.s })\end{array}$ & $\begin{array}{l}\text { PM RA } \\
\text { (mas) }\end{array}$ & $\begin{array}{l}\text { PM Dec } \\
\text { (mas) }\end{array}$ & $\begin{array}{c}\mathrm{RA}+\mathrm{PM} \\
(\mathrm{hh} \mathrm{mm} \mathrm{ss} . \mathrm{ss})\end{array}$ & $\begin{array}{c}\text { Dec+PM } \\
\text { (dd mm ss.s) }\end{array}$ \\
\hline DP Leo & $\mathrm{AM}$ & 111715.93 & +175742.0 & & & 111715.93 & +175742.0 \\
\hline DO Dra & IP & 114338.50 & +714120.7 & 16.9 & 10.9 & 114338.52 & +714120.9 \\
\hline EU UMa & $\mathrm{AM}$ & 114955.71 & +284507.6 & & & 114955.71 & +284507.6 \\
\hline EV UMa & $\mathrm{AM}$ & 130753.87 & +535130.6 & & & 130753.87 & +535130.6 \\
\hline Vir 1 & $\mathrm{AM}$ & 142256.32 & -022108.0 & & & 142256.32 & -022108.0 \\
\hline Vir 2 & $\mathrm{AM}$ & 142438.93 & -022739.3 & & & 142438.93 & -02 2739.3 \\
\hline $\mathrm{BM}$ CrB & $\mathrm{AM}$ & 154104.67 & +360252.9 & & & 154104.67 & +360252.9 \\
\hline MR Ser & $\mathrm{AM}$ & 155247.18 & +185629.2 & 11 & 51.7 & 155247.19 & +185629.9 \\
\hline $\mathrm{AP} \mathrm{CrB}$ & $\mathrm{AM}$ & 155412.34 & +272152.4 & -18 & 24 & 155412.32 & +272152.8 \\
\hline V519 Ser & $\mathrm{AM}$ & 161007.51 & +035233.0 & & & 161007.51 & +035233.0 \\
\hline Her 1 & $\mathrm{AM}$ & 162608.16 & +332827.8 & & & 162608.16 & +332827.8 \\
\hline V1189 Her & $\mathrm{AM}$ & 162936.53 & +263519.6 & -12 & -6 & 162936.52 & +263519.5 \\
\hline V1237 Her & $\mathrm{AM}$ & 170053.30 & +400357.7 & & & 170053.30 & +400357.7 \\
\hline V795 Her & IP & 171256.17 & +33 3119.2 & 3 & -23.7 & 171256.17 & +33 3118.9 \\
\hline V1007 Her & $\mathrm{AM}$ & 172406.28 & +411408.0 & -2.4 & 3.2 & 172406.28 & +411408.0 \\
\hline V2731 Oph & IP & 173021.90 & -055932.1 & 1 & 12.9 & 173021.90 & -055931.9 \\
\hline Sgr 1 & $\mathrm{AM}$ & 174532.70 & -290552.0 & 0.3 & -0.6 & 174532.70 & -290552.0 \\
\hline V2301 Oph & $\mathrm{AM}$ & 180035.58 & +08 1013.6 & & & 180035.58 & +08 1013.6 \\
\hline V884 Her & $\mathrm{AM}$ & 180206.52 & +180444.9 & -46.8 & -75.5 & 180206.48 & +180443.9 \\
\hline V1323 Her & IP & 180339.67 & +401220.6 & 1.3 & -1.8 & 180339.67 & +401220.6 \\
\hline DQ Her & IP & 180730.26 & +455132.1 & 5.3 & 16.7 & 180730.26 & +455132.3 \\
\hline V426 Oph & IP ? & 180751.69 & +055147.8 & 1.7 & -34.4 & 180751.69 & +055147.3 \\
\hline AM Her & $\mathrm{AM}$ & 181613.33 & +495204.3 & -46.3 & 27 & $18 \quad 16 \quad 13.29$ & +495204.7 \\
\hline Sct 1 & IP ? & 183220.00 & -084030.0 & -12.1 & 3.8 & 183219.99 & -08 4029.9 \\
\hline MT Dra & $\mathrm{AM}$ & 184658.83 & +553829.1 & -9.9 & -8.8 & 184658.82 & +553829.0 \\
\hline V603 Aql & $\mathrm{SH} ?$ & 184854.64 & +003502.9 & 10.8 & -8.7 & 184854.65 & +003502.8 \\
\hline V373 Sct & IP ? & 185526.71 & -074305.5 & & & 185526.71 & -074305.5 \\
\hline V1425 Aql & IP ? & 190526.68 & -014214.2 & 3.5 & 4.4 & 190526.68 & -014214.1 \\
\hline EP Dra & $\mathrm{AM}$ & 190706.18 & +690844.2 & -1.2 & -10.3 & 190706.18 & +690844.0 \\
\hline V407 Vul & $\mathrm{AM} ?$ & 191426.07 & +245643.3 & -9.9 & 0.7 & 191426.06 & +245643.3 \\
\hline V1432 Aql & $\mathrm{AM}$ & 194011.42 & -102525.8 & -15.2 & -3.5 & 194011.41 & -102525.8 \\
\hline V2306 Cyg & IP & 195814.48 & +323242.2 & 0.9 & 2 & 195814.48 & +323242.2 \\
\hline QQ Vul & $\mathrm{AM}$ & 200541.91 & +223958.9 & 9.2 & -8.2 & 200541.92 & +223958.8 \\
\hline WZ Sge & WZ ? & 200736.41 & +174215.4 & 102 & -10 & 200736.50 & +174215.3 \\
\hline V4738 Sgr & $\mathrm{AM}$ & 202237.53 & -395412.8 & & & 202237.53 & -395412.8 \\
\hline AE Aqr & IP & 204009.16 & -005215.1 & 68.3 & 14.3 & $2040 \quad 09.23$ & +005214.9 \\
\hline Aqr 1 & $\mathrm{AM}$ & 204827.91 & +005008.9 & 5.9 & 3.4 & 204827.92 & +005009.0 \\
\hline HU Aqr & $\mathrm{AM}$ & 210758.22 & $-05 \quad 1740.1$ & -61.9 & -55.1 & 210758.16 & -051740.9 \\
\hline V1500 Cyg & $\mathrm{AM}$ & 211136.51 & +480902.8 & 8.1 & -2.6 & 211136.52 & +480902.8 \\
\hline V2069 Cyg & IP & 212344.84 & +421801.8 & -1.7 & -9.6 & 212344.84 & +421801.7 \\
\hline Cyg 1 & IP & 213343.65 & +510724.5 & -2 & 4.5 & 213343.65 & +510724.6 \\
\hline LS Peg & IP & 215157.93 & +140653.3 & 17.9 & -15.7 & 215157.95 & +140653.1 \\
\hline
\end{tabular}


JVLA MCV SuRvey Data

Table 2 (continued)

\begin{tabular}{llrrrrrr}
\hline \hline GCVS Name & Class $^{\mathrm{a}}$ & \multicolumn{1}{c}{ RA } & \multicolumn{1}{c}{$\begin{array}{c}\text { Dec } \\
\text { (hh mm ss.ss) }\end{array}$} & $\begin{array}{c}\text { PM RA } \\
\text { (dd mm ss.s) }\end{array}$ & $\begin{array}{c}\text { PM Dec } \\
\text { (mas) }\end{array}$ & $\begin{array}{c}\text { RA+PM } \\
\text { (hh mm ss.ss) }\end{array}$ & $\begin{array}{c}\text { Dec+PM } \\
\text { (dd mm ss.s) }\end{array}$ \\
\hline V388 Peg & AM & 215732.30 & +085515.4 & -1.5 & -1.6 & 215732.30 & +085515.4 \\
FO Aqr & IP & 221755.39 & -082103.8 & 4.1 & 1.6 & 221755.39 & -082103.8 \\
Aqr 2 & IP ? & 223843.83 & +010820.7 & -20.6 & -0.3 & 223843.81 & +010820.7 \\
AO Psc & IP & 225517.99 & -031040.0 & 9.3 & -20.5 & 225518.00 & -031040.3 \\
Aqr 5 & AM & 231603.65 & -052708.7 & -4.4 & -18.1 & 231603.65 & -052709.0 \\
BW Scl & WZ & 235300.82 & -385146.0 & 74.7 & -58.7 & 235300.90 & -385146.9 \\
\hline
\end{tabular}

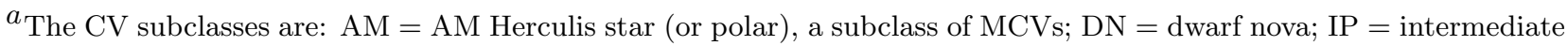
polar and DQ Herculis star, subclasses of MCVs; SH = non-SU UMa star showing permanent or transient superhumps, a subclass of nova-like (NL) CVs; SU = SU Ursa Majoris star, a subtype of DN; SW = SW Sextans star, a subtype of NLs; VY = VY Sculptoris star, a subclass of NLs; WZ = WZ Sagittae star, a subclass of SU UMa stars; ? = uncertain classification.

Table 3 is the list of 19 detected MCVs. As in Table 1 , columns 1 and 2 are the GCVS name and semester scheduling-block designation. These two values uniquely determine the observation. Columns 3 and 4 are the observed source position. For stars with detections at multiple frequencies, the most accurate position is given, which equates to the measured position of the highest frequency observation. Column 5 is the distance between the observed and cataloged source positions and its standard deviation is the approximate size of the longest axis of the synthesized beam at half-width-halfmaximum. Columns $6-8$ are respectively the detected flux and error at the C-, X-, and K-band frequencies.
Column 9 is the significance of the detection, represented as a signal-to-noise $(\mathrm{S} / \mathrm{N})$ value. The significance of each observation is a combination of the source position and flux densities. Six of the new detections (Cas 1, BS Tri, UZ For, WX LMi, BM CrB, and Her 1) have no measured proper motion, so their cataloged position is for epoch J2000 and not for the epoch of the observation (i.e., circa J2014). This likely explains the discrepancy between the observed and catalog positions for some of these stars. As for V1007 Her, AM Her, and QQ Vul that have proper motions, we currently have no explanation for the large discrepancy in the observed and cataloged positions.

Table 3. Radio fluxes and positions of detected MCVs

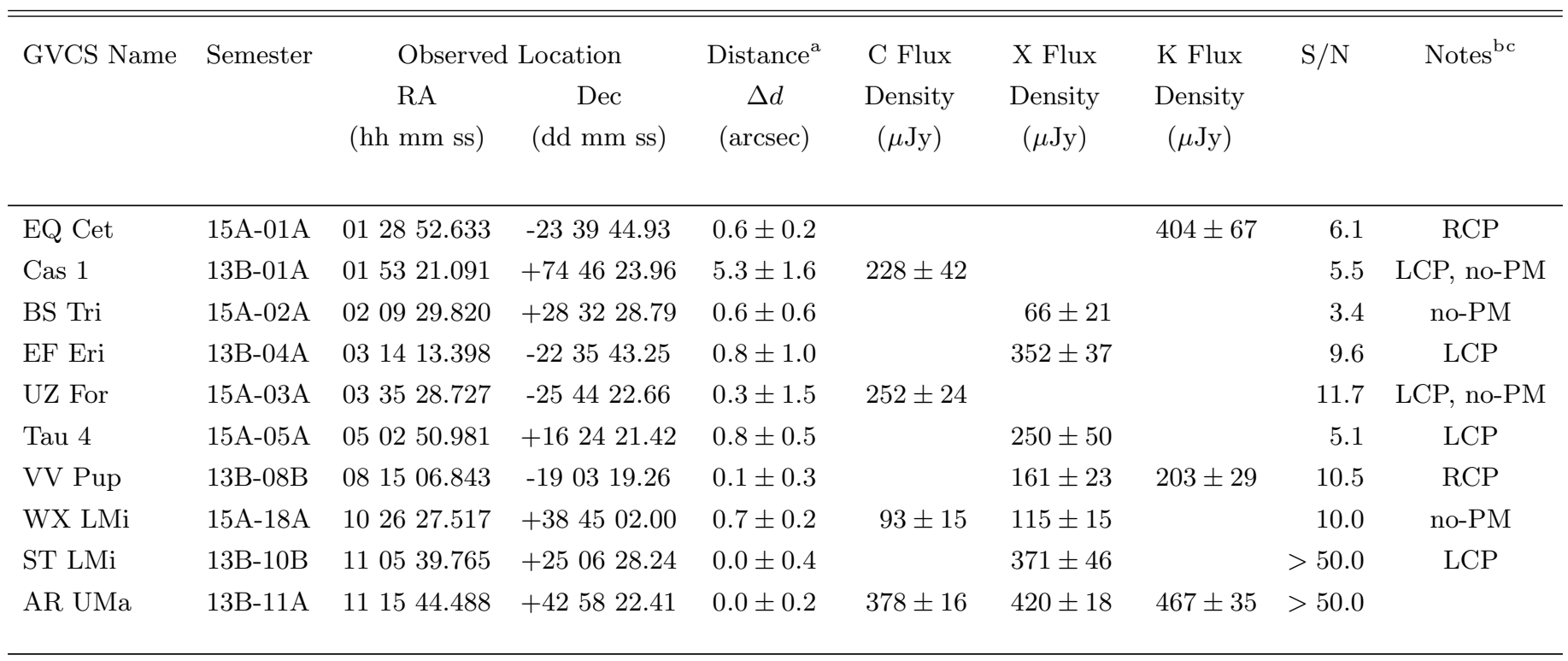


Table 3 (continued)

\begin{tabular}{|c|c|c|c|c|c|c|c|c|c|}
\hline \multirow[t]{2}{*}{ GVCS Name } & \multirow[t]{2}{*}{ Semester } & \multicolumn{2}{|c|}{ Observed Location } & \multirow{2}{*}{$\begin{array}{c}\text { Distance }^{\mathrm{a}} \\
\Delta d \\
(\operatorname{arcsec})\end{array}$} & \multirow{2}{*}{$\begin{array}{c}\text { C Flux } \\
\text { Density } \\
(\mu \mathrm{Jy})\end{array}$} & \multirow{2}{*}{$\begin{array}{c}\text { X Flux } \\
\text { Density } \\
(\mu \mathrm{Jy})\end{array}$} & \multirow{2}{*}{$\begin{array}{c}\text { K Flux } \\
\text { Density } \\
(\mu \mathrm{Jy})\end{array}$} & \multirow[t]{2}{*}{$\mathrm{S} / \mathrm{N}$} & \multirow[t]{2}{*}{ Notes $^{\text {bc }}$} \\
\hline & & $\begin{array}{c}\mathrm{RA} \\
(\mathrm{hh} \mathrm{mm} \mathrm{ss})\end{array}$ & $\begin{array}{c}\text { Dec } \\
(\mathrm{dd} \mathrm{mm} \mathrm{ss})\end{array}$ & & & & & & \\
\hline AR UMa & 13B-11B & 111544.487 & +425822.41 & $0.0 \pm 0.2$ & $539 \pm 22$ & $461 \pm 18$ & $352 \pm 33$ & $>50.0$ & \\
\hline $\mathrm{BM} \mathrm{CrB}$ & 13B-13A & 154104.666 & +360252.69 & $0.2 \pm 0.4$ & & $158 \pm 28$ & & 6.1 & LCP, no-PM \\
\hline MR Ser & 13B-13A & 155247.153 & +185629.68 & $0.5 \pm 0.4$ & $603 \pm 35$ & $424 \pm 27$ & & 23.6 & $\mathrm{RCP}$ \\
\hline Her 1 & $15 \mathrm{~A}-24 \mathrm{~A}$ & $1626 \quad 08.212$ & +332827.00 & $1.1 \pm 0.2$ & & & $218 \pm 22$ & 9.9 & RCP, no-PM \\
\hline V1007 Her & $15 \mathrm{~A}-25 \mathrm{~A}$ & 172406.299 & +411409.97 & $2.0 \pm 0.8$ & & $165 \pm 25$ & & 6.6 & $\mathrm{RCP}$ \\
\hline V1323 Her & $15 \mathrm{~A}-25 \mathrm{~A}$ & 180339.666 & +401219.61 & $1.0 \pm 0.6$ & & $147 \pm 23$ & & 6.5 & LCP \\
\hline V1323 Her & $15 \mathrm{~A}-25 \mathrm{~B}$ & 180339.545 & +401218.71 & $2.8 \pm 1.4$ & $121 \pm 20$ & & & 6.1 & $\mathrm{RCP}$ \\
\hline AM Her & $13 \mathrm{~B}-15 \mathrm{~A}$ & 181613.187 & +495205.29 & $1.8 \pm 1.0$ & $114 \pm 12$ & & & 9.5 & \\
\hline AM Her & 13B-15B & 181613.186 & +495205.15 & $1.4 \pm 0.4$ & $154 \pm 10$ & $243 \pm 10$ & $818 \pm 92$ & $>50.0$ & \\
\hline V603 Aql & $13 \mathrm{~B}-17 \mathrm{~A}$ & 184854.647 & +003502.88 & $0.3 \pm 1.5$ & & $59 \pm 19$ & & 6.0 & $\mathrm{LCP}$ \\
\hline V603 Aql & 13B-17B & 184854.668 & +003503.01 & $0.1 \pm 1.4$ & $47 \pm 8$ & $88 \pm 8$ & & 18.9 & $\mathrm{RCP}$ \\
\hline QQ Vul & 13B-18B & 200541.901 & +224001.89 & $3.1 \pm 0.2$ & & & $438 \pm 43$ & 10.2 & LCP \\
\hline $\mathrm{AE}$ Aqr & $15 \mathrm{~A}-30 \mathrm{~A}$ & 204009.231 & -005214.86 & $0.0 \pm 0.2$ & $5000 \pm 22$ & $5472 \pm 18$ & $8354 \pm 38$ & $>50.0$ & \\
\hline AE Aqr & $15 \mathrm{~A}-30 \mathrm{~B}$ & $2040 \quad 09.233$ & -005214.86 & $0.0 \pm 0.2$ & $5041 \pm 20$ & $5211 \pm 16$ & $4771 \pm 30$ & $>50.0$ & \\
\hline
\end{tabular}

$a^{a} d$ is the distance between the observed and cataloged source positions and its standard deviation is the approximate size of the longest axis of the synthesize beam at half-width-half-maximum.

${ }^{b} \mathrm{LCP}$ and RCP denotes that the detection is seen in only the left-hand or right-hand circular polarization channel.

${ }^{c}$ no-PM denotes that the source has no known proper motion. Therefore, the distance between the observed and cataloged positions may be large.

To estimate the probability that an unrelated extragalactic background radio source close to the stellar position may lead to a false detection, we use the radio source counts statistics of Condon et al. (2012). At an observing frequency of $3 \mathrm{GHz}$ the number of extragalactic backgrounds sources above $100 \mu \mathrm{Jy}$ is approximately 1.15 million per steradian. This corresponds to an areal density of $2.7 \times 10^{-5}$ per square arcsecond, i.e. the chances of a unrelated background source above $100 \mu \mathrm{Jy}$ being within a radius of an arcsecond of our stellar positions is extremely low, approximately 1 in 12,000 . Assuming a standard spectral index for the background sources falling as spectral index $\nu^{-0.6}$, and ignoring unrelated phenomena such as interferometry resolution effects and source variability, we can also derive the chances of a confusing source within 1 arcsecond radius from the stellar positions above $100 \mu \mathrm{Jy}$ at 8 and $22 \mathrm{GHz}$ ( 1 in 21,000 and 1 in 37,000, respectively).

These statistics indicate that extragalactic background sources are unlikely to contaminate our stellar radio detections. Systematic effects such as grating sidelobes from nearby sources distributing appar- ent radio flux across our detection images, and the effects of extended thermal and non-thermal emission in some directions close to the Galactic plane, can lead to apparent radio emission from our stellar positions for some JVLA configurations. In these situations additional tests (seeking point sources, including CLEANing larger fields to remove sidelobes, comparing tentative stellar emission detections amongst different intermediate frequency and polarization channels, and developing an understanding of the regions our stellar sources are located in) can be used to develop confidence in a detection.

Table 4 lists the maximum flux density and its standard deviation for all observations, i.e., for detections and non-detections. A region having a diameter of 4 arcseconds and centered on the expected source location is used unless the observation contains a detection. In that case a background region not affected by any radio sources was used. Columns 1 and 2 are the same as those in Table 3. Columns $3-8$ are the maximum flux density and its standard deviation for the C-, X-, and K-bands, respectively. For nondetections, Table 4 
JVLA MCV SuRvey Data

provides an estimate of the flux density upper limit.

Table 4. Image Statistics

\begin{tabular}{lrrrr}
\hline \hline GCVS Name & Semester & \multicolumn{1}{c}{ C Flux } \\
Density \\
\end{tabular}


BARRETT ET AL.

Table 4 (continued)

\begin{tabular}{|c|c|c|c|c|}
\hline GCVS Name & Semester & $\begin{array}{c}\text { C Flux } \\
\text { Density } \\
(\mu \mathrm{Jy} / \text { beam })\end{array}$ & $\begin{array}{c}\text { X Flux } \\
\text { Density } \\
(\mu \mathrm{Jy} / \text { beam })\end{array}$ & $\begin{array}{c}\text { K Flux } \\
\text { Density } \\
(\mu \mathrm{Jy} / \text { beam })\end{array}$ \\
\hline Tau 4 & $15 \mathrm{~A}-05 \mathrm{~A}$ & $0 \pm 218$ & $131 \pm 57$ & $94 \pm 32$ \\
\hline Tau 4 & $15 \mathrm{~A}-05 \mathrm{~B}$ & $59 \pm 79$ & $99 \pm 37$ & $170 \pm 46$ \\
\hline V1062 Tau & $15 \mathrm{~A}-05 \mathrm{~A}$ & $42 \pm 32$ & $11 \pm 22$ & $90 \pm 24$ \\
\hline V1062 Tau & $15 \mathrm{~A}-05 \mathrm{~B}$ & $0 \pm 56$ & $52 \pm 16$ & $134 \pm 42$ \\
\hline UU Col & $15 \mathrm{~A}-06 \mathrm{~A}$ & $25 \pm 9$ & $36 \pm 14$ & $96 \pm 34$ \\
\hline UU Col & $15 \mathrm{~A}-06 \mathrm{~B}$ & $55 \pm 18$ & $38 \pm 14$ & $339 \pm 93$ \\
\hline V1309 Ori & 13B-02A & $100 \pm 58$ & $47 \pm 16$ & $169 \pm 75$ \\
\hline V1309 Ori & 13B-02B & $107 \pm 63$ & $66 \pm 27$ & $327 \pm 84$ \\
\hline Aur (Paloma) & $15 \mathrm{~A}-07 \mathrm{~A}$ & $40 \pm 17$ & $45 \pm 15$ & $164 \pm 37$ \\
\hline Aur (Paloma) & $15 \mathrm{~A}-07 \mathrm{~B}$ & $29 \pm 12$ & $46 \pm 11$ & $90 \pm 26$ \\
\hline TV Col & 13B-05A & $77 \pm 60$ & $107 \pm 41$ & $233 \pm 96$ \\
\hline TV Col & 13B-05B & $112 \pm 52$ & $94 \pm 57$ & $217 \pm 70$ \\
\hline BY Cam & 13B-01A & $75 \pm 31$ & $66 \pm 25$ & $333 \pm 77$ \\
\hline BY Cam & 13B-06A & $82 \pm 32$ & $49 \pm 24$ & $236 \pm 83$ \\
\hline BY Cam & 13B-01B & $38 \pm 18$ & $38 \pm 18$ & $97 \pm 31$ \\
\hline BY Cam & 13B-06B & $56 \pm 22$ & & $96 \pm 24$ \\
\hline V405 Aur & 13B-06A & $340 \pm 289$ & $145 \pm 67$ & $319 \pm 114$ \\
\hline V405 Aur & 13B-06B & $91 \pm 99$ & & $90 \pm 27$ \\
\hline LS Cam & $15 \mathrm{~A}-08 \mathrm{~A}$ & $40 \pm 22$ & $33 \pm 11$ & $51 \pm 13$ \\
\hline LS Cam & $15 \mathrm{~A}-08 \mathrm{~B}$ & $45 \pm 20$ & $31 \pm 11$ & $80 \pm 23$ \\
\hline Lep 1 & $15 \mathrm{~A}-06 \mathrm{~A}$ & $19 \pm 21$ & $28 \pm 14$ & $132 \pm 31$ \\
\hline Lep 1 & $15 \mathrm{~A}-06 \mathrm{~B}$ & $55 \pm 19$ & $44 \pm 15$ & $340 \pm 90$ \\
\hline V552 Aur & 13B-07A & $15 \pm 24$ & $63 \pm 25$ & $188 \pm 66$ \\
\hline V552 Aur & 13B-07B & $62 \pm 23$ & $41 \pm 18$ & $154 \pm 33$ \\
\hline MU Cam & 13B-06A & $75 \pm 27$ & $65 \pm 22$ & $273 \pm 96$ \\
\hline MU Cam & 13B-06B & & & $88 \pm 28$ \\
\hline V647 Aur & $15 \mathrm{~A}-07 \mathrm{~A}$ & $39 \pm 22$ & $47 \pm 16$ & $178 \pm 49$ \\
\hline V647 Aur & 15A-07B & $23 \pm 19$ & $37 \pm 11$ & $87 \pm 29$ \\
\hline Mon 1 & $15 \mathrm{~A}-04 \mathrm{~A}$ & $64 \pm 24$ & $49 \pm 15$ & $229 \pm 63$ \\
\hline Mon 1 & $15 \mathrm{~A}-04 \mathrm{~B}$ & $51 \pm 20$ & $48 \pm 15$ & $307 \pm 86$ \\
\hline V418 Gem & $15 \mathrm{~A}-09 \mathrm{~A}$ & $31 \pm 15$ & $39 \pm 16$ & $232 \pm 43$ \\
\hline V418 Gem & 15A-09B & $36 \pm 14$ & $33 \pm 12$ & \\
\hline LW Cam & $15 \mathrm{~A}-10 \mathrm{~A}$ & $23 \pm 16$ & $29 \pm 13$ & $57 \pm 22$ \\
\hline LW Cam & $15 \mathrm{~A}-10 \mathrm{~B}$ & $34 \pm 21$ & $43 \pm 15$ & $457 \pm 141$ \\
\hline V348 Pup & 13B-05A & $29 \pm 25$ & $27 \pm 20$ & $185 \pm 66$ \\
\hline V348 Pup & 13B-05B & $50 \pm 26$ & $68 \pm 23$ & $201 \pm 67$ \\
\hline HS Cam & $15 \mathrm{~A}-08 \mathrm{~A}$ & $30 \pm 12$ & $27 \pm 12$ & $59 \pm 16$ \\
\hline HS Cam & $15 \mathrm{~A}-08 \mathrm{~B}$ & $30 \pm 12$ & $32 \pm 12$ & $57 \pm 20$ \\
\hline GI Mon & 13B-08A & $53 \pm 21$ & $47 \pm 17$ & $90 \pm 28$ \\
\hline GI Mon & 13B-08B & $53 \pm 17$ & $56 \pm 20$ & $86 \pm 29$ \\
\hline
\end{tabular}


JVLA MCV SuRvey Data

Table 4 (continued)

\begin{tabular}{|c|c|c|c|c|}
\hline GCVS Name & Semester & $\begin{array}{c}\text { C Flux } \\
\text { Density } \\
(\mu \mathrm{Jy} / \text { beam })\end{array}$ & $\begin{array}{c}\text { X Flux } \\
\text { Density } \\
(\mu \mathrm{Jy} / \text { beam })\end{array}$ & $\begin{array}{c}\text { K Flux } \\
\text { Density } \\
(\mu \mathrm{Jy} / \text { beam })\end{array}$ \\
\hline BG Cmi & 13B-09A & $29 \pm 21$ & $23 \pm 17$ & $181 \pm 81$ \\
\hline BG Cmi & 13B-09B & $55 \pm 17$ & $53 \pm 20$ & $131 \pm 35$ \\
\hline Mon 2 & $15 \mathrm{~A}-11 \mathrm{~A}$ & & $51 \pm 21$ & $103 \pm 27$ \\
\hline Mon 2 & $15 \mathrm{~A}-11 \mathrm{~B}$ & $43 \pm 15$ & $33 \pm 11$ & $93 \pm 27$ \\
\hline PQ Gem & 13B-09A & $41 \pm 31$ & $57 \pm 24$ & $185 \pm 47$ \\
\hline PQ Gem & 13B-09B & $29 \pm 14$ & $49 \pm 22$ & $125 \pm 33$ \\
\hline EU Lyn & $15 \mathrm{~A}-09 \mathrm{~A}$ & $34 \pm 17$ & $48 \pm 11$ & $224 \pm 51$ \\
\hline EU Lyn & $15 \mathrm{~A}-09 \mathrm{~B}$ & $39 \pm 14$ & $32 \pm 9$ & \\
\hline EV Lyn & $15 \mathrm{~A}-12 \mathrm{~A}$ & $28 \pm 21$ & $28 \pm 11$ & $69 \pm 25$ \\
\hline EV Lyn & $15 \mathrm{~A}-12 \mathrm{~B}$ & $21 \pm 15$ & $17 \pm 9$ & $75 \pm 25$ \\
\hline HT Cam & $15 \mathrm{~A}-10 \mathrm{~A}$ & $17 \pm 9$ & $33 \pm 13$ & $69 \pm 22$ \\
\hline HT Cam & $15 \mathrm{~A}-10 \mathrm{~B}$ & $31 \pm 16$ & $25 \pm 14$ & $89 \pm 24$ \\
\hline DW Cnc & $15 \mathrm{~A}-13 \mathrm{~A}$ & $57 \pm 26$ & $46 \pm 16$ & $204 \pm 59$ \\
\hline DW Cnc & $15 \mathrm{~A}-13 \mathrm{~B}$ & $66 \pm 27$ & $51 \pm 19$ & $270 \pm 72$ \\
\hline Cnc 1 & $15 \mathrm{~A}-13 \mathrm{~A}$ & $41 \pm 15$ & $52 \pm 16$ & $235 \pm 60$ \\
\hline Cnc 1 & $15 \mathrm{~A}-13 \mathrm{~B}$ & $53 \pm 16$ & $44 \pm 15$ & $405 \pm 88$ \\
\hline V351 Pup & $13 \mathrm{~B}-05 \mathrm{~A}$ & $45 \pm 22$ & $60 \pm 21$ & $150 \pm 54$ \\
\hline V351 Pup & 13B-05B & $33 \pm 17$ & $43 \pm 20$ & $154 \pm 48$ \\
\hline VV Pup & 13B-08A & $60 \pm 23$ & $58 \pm 26$ & $159 \pm 36$ \\
\hline VV Pup & 13B-08B & $78 \pm 33$ & $44 \pm 21$ & $114 \pm 35$ \\
\hline EG Lyn & $15 \mathrm{~A}-12 \mathrm{~A}$ & $22 \pm 12$ & $34 \pm 12$ & $63 \pm 23$ \\
\hline EG Lyn & $15 \mathrm{~A}-12 \mathrm{~B}$ & $42 \pm 16$ & $28 \pm 12$ & $73 \pm 21$ \\
\hline WX Pyx & $15 \mathrm{~A}-11 \mathrm{~A}$ & & $46 \pm 17$ & $162 \pm 42$ \\
\hline WX Pyx & $15 \mathrm{~A}-11 \mathrm{~B}$ & $46 \pm 13$ & $57 \pm 13$ & $141 \pm 43$ \\
\hline SW Uma & 13B-07A & $0 \pm 38$ & $51 \pm 28$ & $141 \pm 50$ \\
\hline SW Uma & 13B-07B & $47 \pm 17$ & $48 \pm 15$ & $102 \pm 31$ \\
\hline Lyn 1 & $15 \mathrm{~A}-14 \mathrm{~A}$ & $26 \pm 16$ & $50 \pm 19$ & $63 \pm 16$ \\
\hline Lyn 1 & $15 \mathrm{~A}-14 \mathrm{~B}$ & $48 \pm 14$ & $32 \pm 10$ & $139 \pm 31$ \\
\hline Cnc 2 & $15 \mathrm{~A}-14 \mathrm{~A}$ & $24 \pm 17$ & $43 \pm 19$ & $54 \pm 15$ \\
\hline Cnc 2 & $15 \mathrm{~A}-14 \mathrm{~B}$ & $40 \pm 13$ & $36 \pm 12$ & $130 \pm 31$ \\
\hline EU Cnc & $15 \mathrm{~A}-15 \mathrm{~A}$ & $40 \pm 13$ & $60 \pm 15$ & $262 \pm 62$ \\
\hline EU Cnc & $15 \mathrm{~A}-15 \mathrm{~B}$ & $39 \pm 21$ & $48 \pm 15$ & $249 \pm 79$ \\
\hline Hya 1 & 13B-09A & $182 \pm 93$ & $50 \pm 29$ & $216 \pm 78$ \\
\hline Hya 1 & 13B-09B & $39 \pm 44$ & $55 \pm 27$ & $127 \pm 36$ \\
\hline GZ Cnc & $15 \mathrm{~A}-16 \mathrm{~A}$ & $61 \pm 21$ & $53 \pm 15$ & $212 \pm 60$ \\
\hline GZ Cnc & $15 \mathrm{~A}-16 \mathrm{~B}$ & $40 \pm 14$ & $56 \pm 16$ & $251 \pm 67$ \\
\hline HU Leo & $15 \mathrm{~A}-16 \mathrm{~A}$ & $45 \pm 14$ & $52 \pm 16$ & $192 \pm 52$ \\
\hline HU Leo & $15 \mathrm{~A}-16 \mathrm{~B}$ & $44 \pm 14$ & $47 \pm 13$ & $210 \pm 54$ \\
\hline HS $0922+1333$ & $15 \mathrm{~A}-15 \mathrm{~A}$ & $36 \pm 15$ & $30 \pm 14$ & $314 \pm 79$ \\
\hline HS $0922+1333$ & $15 \mathrm{~A}-15 \mathrm{~B}$ & $44 \pm 16$ & $31 \pm 16$ & $289 \pm 80$ \\
\hline
\end{tabular}


BARRETT ET AL.

Table 4 (continued)

\begin{tabular}{|c|c|c|c|c|}
\hline GCVS Name & Semester & $\begin{array}{c}\text { C Flux } \\
\text { Density } \\
(\mu \mathrm{Jy} / \text { beam })\end{array}$ & $\begin{array}{c}\text { X Flux } \\
\text { Density } \\
(\mu \mathrm{Jy} / \text { beam })\end{array}$ & $\begin{array}{c}\text { K Flux } \\
\text { Density } \\
(\mu \mathrm{Jy} / \text { beam })\end{array}$ \\
\hline MN Hya & $15 \mathrm{~A}-17 \mathrm{~A}$ & $58 \pm 18$ & $51 \pm 15$ & $274 \pm 78$ \\
\hline MN Hya & $15 \mathrm{~A}-17 \mathrm{~B}$ & $47 \pm 19$ & $42 \pm 15$ & $336 \pm 71$ \\
\hline US 691 & $15 \mathrm{~A}-18 \mathrm{~A}$ & $28 \pm 13$ & $43 \pm 13$ & $65 \pm 46$ \\
\hline VZ Sex & $15 \mathrm{~A}-19 \mathrm{~A}$ & $16 \pm 12$ & $41 \pm 12$ & $68 \pm 26$ \\
\hline VZ Sex & 15A-19B & $23 \pm 26$ & $36 \pm 15$ & $101 \pm 37$ \\
\hline HY Leo & $15 \mathrm{~A}-20 \mathrm{~A}$ & $60 \pm 31$ & $42 \pm 11$ & $92 \pm 24$ \\
\hline HY Leo & $15 \mathrm{~A}-20 \mathrm{~B}$ & $42 \pm 17$ & $45 \pm 15$ & $79 \pm 19$ \\
\hline Leo 1 & $15 \mathrm{~A}-20 \mathrm{~A}$ & $48 \pm 23$ & $40 \pm 14$ & $87 \pm 24$ \\
\hline Leo 1 & $15 \mathrm{~A}-20 \mathrm{~B}$ & $33 \pm 14$ & $45 \pm 13$ & $74 \pm 18$ \\
\hline Hya 2 & 13B-08A & $20 \pm 41$ & $61 \pm 22$ & $144 \pm 41$ \\
\hline Hya 2 & 13B-08B & $17 \pm 33$ & $37 \pm 22$ & $127 \pm 40$ \\
\hline Hya 3 & $15 \mathrm{~A}-17 \mathrm{~A}$ & $28 \pm 33$ & $70 \pm 22$ & $372 \pm 87$ \\
\hline Hya 3 & 15A-17B & $47 \pm 23$ & $63 \pm 25$ & $262 \pm 76$ \\
\hline GG Leo & 13B-10A & $63 \pm 26$ & $70 \pm 26$ & $112 \pm 41$ \\
\hline GG Leo & 13B-10B & $66 \pm 25$ & $61 \pm 22$ & $175 \pm 45$ \\
\hline WX Lmi & $15 \mathrm{~A}-18 \mathrm{~A}$ & $91 \pm 17$ & $90 \pm 17$ & $133 \pm 37$ \\
\hline YY Sex & 15A-19A & $18 \pm 17$ & $35 \pm 16$ & $87 \pm 24$ \\
\hline YY Sex & 15A-19B & $7 \pm 19$ & $32 \pm 17$ & $108 \pm 33$ \\
\hline FH Uma & $15 \mathrm{~A}-21 \mathrm{~A}$ & $37 \pm 14$ & $34 \pm 14$ & $72 \pm 18$ \\
\hline FH Uma & $15 \mathrm{~A}-21 \mathrm{~B}$ & $30 \pm 15$ & $28 \pm 11$ & $84 \pm 20$ \\
\hline EK Uma & $15 \mathrm{~A}-21 \mathrm{~A}$ & $47 \pm 16$ & $34 \pm 13$ & $61 \pm 16$ \\
\hline EK Uma & $15 \mathrm{~A}-21 \mathrm{~B}$ & $42 \pm 10$ & $29 \pm 11$ & $61 \pm 19$ \\
\hline AN Uma & 13B-11A & $40 \pm 19$ & $61 \pm 20$ & $117 \pm 32$ \\
\hline AN Uma & 13B-11B & $63 \pm 23$ & $50 \pm 16$ & $122 \pm 37$ \\
\hline ST Lmi & 13B-10A & $48 \pm 24$ & $89 \pm 38$ & $134 \pm 37$ \\
\hline ST Lmi & 13B-10B & $50 \pm 23$ & $51 \pm 17$ & $146 \pm 39$ \\
\hline AR Uma & 13B-11A & $378 \pm 16$ & $420 \pm 18$ & $467 \pm 35$ \\
\hline AR Uma & 13B-11B & $539 \pm 22$ & $461 \pm 18$ & $352 \pm 33$ \\
\hline DP Leo & 13B-10A & $89 \pm 34$ & $61 \pm 25$ & $143 \pm 41$ \\
\hline DP Leo & 13B-10B & $55 \pm 29$ & $50 \pm 21$ & $139 \pm 47$ \\
\hline DO Dra & 13B-07A & $54 \pm 22$ & $38 \pm 17$ & $308 \pm 70$ \\
\hline DO Dra & 13B-07B & $47 \pm 28$ & $44 \pm 14$ & $103 \pm 33$ \\
\hline EU Uma & $13 \mathrm{~B}-10 \mathrm{~A}$ & $78 \pm 41$ & $78 \pm 26$ & $146 \pm 43$ \\
\hline EU Uma & 13B-10B & $99 \pm 42$ & $63 \pm 29$ & $197 \pm 47$ \\
\hline EV Uma & 13B-11A & $44 \pm 15$ & $57 \pm 18$ & $92 \pm 39$ \\
\hline EV Uma & 13B-11B & $52 \pm 21$ & $47 \pm 18$ & $107 \pm 36$ \\
\hline Vir 1 & $15 \mathrm{~A}-22 \mathrm{~A}$ & $28 \pm 10$ & $29 \pm 10$ & $64 \pm 17$ \\
\hline Vir 1 & $15 \mathrm{~A}-22 \mathrm{~B}$ & $33 \pm 21$ & $38 \pm 16$ & $94 \pm 23$ \\
\hline Vir 2 & $15 \mathrm{~A}-22 \mathrm{~A}$ & $42 \pm 23$ & $33 \pm 13$ & $48 \pm 14$ \\
\hline Vir 2 & $15 \mathrm{~A}-22 \mathrm{~B}$ & $76 \pm 25$ & $39 \pm 15$ & $79 \pm 22$ \\
\hline
\end{tabular}


JVLA MCV SuRvey Data

Table 4 (continued)

\begin{tabular}{|c|c|c|c|c|}
\hline GCVS Name & Semester & $\begin{array}{c}\text { C Flux } \\
\text { Density } \\
(\mu \mathrm{Jy} / \text { beam })\end{array}$ & $\begin{array}{c}\text { X Flux } \\
\text { Density } \\
(\mu \mathrm{Jy} / \text { beam })\end{array}$ & $\begin{array}{c}\text { K Flux } \\
\text { Density } \\
(\mu \mathrm{Jy} / \text { beam })\end{array}$ \\
\hline $\mathrm{BM} \mathrm{CrB}$ & 13B-13A & $62 \pm 28$ & $67 \pm 22$ & $72 \pm 25$ \\
\hline MR Ser & 13B-13A & $274 \pm 23$ & $164 \pm 18$ & $100 \pm 24$ \\
\hline $\mathrm{AP} \mathrm{CrB}$ & $15 \mathrm{~A}-23 \mathrm{~A}$ & $20 \pm 69$ & $40 \pm 11$ & $86 \pm 29$ \\
\hline AP CrB & $15 \mathrm{~A}-23 \mathrm{~B}$ & $0 \pm 81$ & $34 \pm 16$ & $97 \pm 25$ \\
\hline V519 Ser & 13B-13A & $36 \pm 16$ & $43 \pm 17$ & $88 \pm 28$ \\
\hline Her 1 & $15 \mathrm{~A}-24 \mathrm{~A}$ & $40 \pm 13$ & $29 \pm 10$ & $89 \pm 24$ \\
\hline Her 1 & $15 \mathrm{~A}-24 \mathrm{~B}$ & $45 \pm 21$ & $30 \pm 13$ & $73 \pm 20$ \\
\hline V1189 Her & $15 \mathrm{~A}-23 \mathrm{~A}$ & $667 \pm 347$ & $1144 \pm 402$ & $113 \pm 97$ \\
\hline V1189 Her & $15 \mathrm{~A}-23 \mathrm{~B}$ & $597 \pm 241$ & $1032 \pm 417$ & $270 \pm 82$ \\
\hline V1237 Her & $15 \mathrm{~A}-24 \mathrm{~A}$ & $40 \pm 18$ & $33 \pm 12$ & $79 \pm 25$ \\
\hline V1237 Her & $15 \mathrm{~A}-24 \mathrm{~B}$ & $59 \pm 33$ & $34 \pm 12$ & $61 \pm 21$ \\
\hline V795 Her & 13B-15A & $9 \pm 5$ & $115 \pm 48$ & \\
\hline V795 Her & 13B-15B & $14 \pm 8$ & $26 \pm 10$ & $357 \pm 105$ \\
\hline V1007 Her & $15 \mathrm{~A}-25 \mathrm{~A}$ & $32 \pm 15$ & $35 \pm 16$ & $83 \pm 25$ \\
\hline V1007 Her & $15 \mathrm{~A}-25 \mathrm{~B}$ & $28 \pm 12$ & $29 \pm 11$ & $69 \pm 22$ \\
\hline V2731 Oph & $15 \mathrm{~A}-26 \mathrm{~A}$ & $371 \pm 160$ & $357 \pm 141$ & $213 \pm 63$ \\
\hline V2731 Oph & $15 \mathrm{~A}-26 \mathrm{~B}$ & $67 \pm 110$ & $255 \pm 148$ & $220 \pm 78$ \\
\hline Sgr 1 & $15 \mathrm{~A}-26 \mathrm{~A}$ & $3900 \pm 2940$ & $0 \pm 432$ & $3 \pm 119$ \\
\hline Sgr 1 & $15 \mathrm{~A}-26 \mathrm{~B}$ & $3453 \pm 2046$ & $0 \pm 348$ & $66 \pm 120$ \\
\hline V2301 Oph & 13B-16A & $21 \pm 9$ & $40 \pm 16$ & \\
\hline V2301 Oph & 13B-16B & $23 \pm 15$ & $60 \pm 19$ & $1300 \pm 507$ \\
\hline V884 Her & 13B-16A & $43 \pm 24$ & $41 \pm 16$ & \\
\hline V884 Her & 13B-16B & $42 \pm 20$ & $78 \pm 26$ & $1426 \pm 447$ \\
\hline V1323 Her & $15 \mathrm{~A}-25 \mathrm{~A}$ & $42 \pm 18$ & $50 \pm 21$ & \\
\hline V1323 Her & $15 \mathrm{~A}-25 \mathrm{~B}$ & $29 \pm 11$ & $29 \pm 11$ & $102 \pm 21$ \\
\hline V426 Oph & 13B-16A & $369 \pm 154$ & $146 \pm 102$ & \\
\hline V426 Oph & 13B-16B & $121 \pm 99$ & $148 \pm 61$ & $1060 \pm 610$ \\
\hline DQ Her & 13B-15A & $41 \pm 7$ & $128 \pm 50$ & \\
\hline DQ Her & 13B-15B & $23 \pm 12$ & $37 \pm 14$ & $288 \pm 104$ \\
\hline AM Her & 13B-15A & $70 \pm 6$ & $162 \pm 52$ & \\
\hline AM Her & 13B-15B & $104 \pm 6$ & $198 \pm 14$ & $665 \pm 98$ \\
\hline Sct 1 & $15 \mathrm{~A}-27 \mathrm{~A}$ & $229 \pm 175$ & $15 \pm 15$ & $79 \pm 29$ \\
\hline Sct 1 & $15 \mathrm{~A}-27 \mathrm{~B}$ & $76 \pm 36$ & $45 \pm 15$ & $80 \pm 22$ \\
\hline MT Dra & $15 \mathrm{~A}-28 \mathrm{~A}$ & $27 \pm 17$ & $22 \pm 10$ & $55 \pm 18$ \\
\hline MT Dra & $15 \mathrm{~A}-28 \mathrm{~B}$ & $37 \pm 20$ & $31 \pm 12$ & $56 \pm 18$ \\
\hline V603 Aql & 13B-17A & $20 \pm 8$ & $44 \pm 17$ & \\
\hline V603 Aql & 13B-17B & $29 \pm 5$ & $57 \pm 12$ & $292 \pm 104$ \\
\hline V373 Sct & 13B-17A & $14 \pm 8$ & $50 \pm 14$ & \\
\hline V373 Sct & 13B-17B & $25 \pm 12$ & $24 \pm 9$ & $429 \pm 119$ \\
\hline V1425 Aql & $15 \mathrm{~A}-27 \mathrm{~A}$ & $55 \pm 23$ & $34 \pm 16$ & $134 \pm 42$ \\
\hline
\end{tabular}


BARRETT ET AL.

Table 4 (continued)

\begin{tabular}{|c|c|c|c|c|}
\hline GCVS Name & Semester & $\begin{array}{c}\text { C Flux } \\
\text { Density } \\
(\mu \mathrm{Jy} / \text { beam })\end{array}$ & $\begin{array}{c}\text { X Flux } \\
\text { Density } \\
(\mu \mathrm{Jy} / \text { beam })\end{array}$ & $\begin{array}{c}\text { K Flux } \\
\text { Density } \\
(\mu \mathrm{Jy} / \text { beam })\end{array}$ \\
\hline V1425 Aql & $15 \mathrm{~A}-27 \mathrm{~B}$ & $40 \pm 15$ & $38 \pm 11$ & $104 \pm 22$ \\
\hline EP Dra & $15 \mathrm{~A}-28 \mathrm{~A}$ & $23 \pm 16$ & $23 \pm 11$ & $57 \pm 17$ \\
\hline EP Dra & $15 \mathrm{~A}-28 \mathrm{~B}$ & $20 \pm 9$ & $31 \pm 14$ & $71 \pm 18$ \\
\hline V407 Vul & $15 \mathrm{~A}-29 \mathrm{~A}$ & $30 \pm 18$ & $30 \pm 12$ & $77 \pm 23$ \\
\hline V407 Vul & $15 \mathrm{~A}-29 \mathrm{~B}$ & $77 \pm 36$ & $33 \pm 13$ & $69 \pm 23$ \\
\hline V1432 Aql & 13B-17A & $9 \pm 5$ & $32 \pm 12$ & \\
\hline V1432 Aql & 13B-17B & $9 \pm 7$ & $26 \pm 6$ & $472 \pm 145$ \\
\hline V2306 Cyg & $15 \mathrm{~A}-29 \mathrm{~A}$ & $22 \pm 12$ & $44 \pm 15$ & $62 \pm 23$ \\
\hline V2306 Cyg & $15 \mathrm{~A}-29 \mathrm{~B}$ & $28 \pm 12$ & $31 \pm 14$ & $55 \pm 18$ \\
\hline QQ Vul & 13B-18A & $76 \pm 34$ & $92 \pm 31$ & $147 \pm 56$ \\
\hline QQ Vul & 13B-18B & $36 \pm 34$ & $40 \pm 17$ & $119 \pm 35$ \\
\hline WZ Sge & 13B-18A & $129 \pm 45$ & $76 \pm 36$ & $147 \pm 48$ \\
\hline WZ Sge & 13B-18B & $63 \pm 30$ & $62 \pm 26$ & $161 \pm 44$ \\
\hline V4738 Sgr & $15 \mathrm{~A}-35 \mathrm{~A}$ & $45 \pm 27$ & $99 \pm 35$ & $195 \pm 55$ \\
\hline V4738 Sgr & $15 \mathrm{~A}-35 \mathrm{~B}$ & $37 \pm 19$ & $68 \pm 37$ & $143 \pm 49$ \\
\hline AE Aqr & $15 \mathrm{~A}-30 \mathrm{~A}$ & $47 \pm 22$ & $51 \pm 18$ & $120 \pm 38$ \\
\hline AE Aqr & $15 \mathrm{~A}-30 \mathrm{~B}$ & $36 \pm 20$ & $61 \pm 16$ & $96 \pm 30$ \\
\hline Aqr 1 & $15 \mathrm{~A}-30 \mathrm{~A}$ & $16 \pm 13$ & $39 \pm 14$ & $47 \pm 16$ \\
\hline Aqr 1 & $15 \mathrm{~A}-30 \mathrm{~B}$ & $41 \pm 17$ & $41 \pm 15$ & $80 \pm 21$ \\
\hline HU Aqr & 13B-19A & $248 \pm 59$ & $179 \pm 77$ & $176 \pm 55$ \\
\hline HU Aqr & 13B-19B & & & $186 \pm 46$ \\
\hline V1500 Cyg & $15 \mathrm{~A}-31 \mathrm{~A}$ & $14 \pm 13$ & $37 \pm 14$ & $53 \pm 18$ \\
\hline V1500 Cyg & $15 \mathrm{~A}-31 \mathrm{~B}$ & $3 \pm 23$ & $39 \pm 14$ & $72 \pm 26$ \\
\hline V2069 Cyg & $15 \mathrm{~A}-31 \mathrm{~A}$ & $34 \pm 12$ & $42 \pm 14$ & $67 \pm 18$ \\
\hline V2069 Cyg & $15 \mathrm{~A}-31 \mathrm{~B}$ & $36 \pm 16$ & $25 \pm 11$ & $82 \pm 22$ \\
\hline Cyg 1 & $15 \mathrm{~A}-32 \mathrm{~A}$ & $56 \pm 15$ & $39 \pm 14$ & $218 \pm 56$ \\
\hline Cyg 1 & $15 \mathrm{~A}-32 \mathrm{~B}$ & $57 \pm 19$ & $46 \pm 13$ & $146 \pm 41$ \\
\hline LS Peg & 13B-18A & $77 \pm 30$ & $59 \pm 25$ & $114 \pm 37$ \\
\hline LS Peg & 13B-18B & $76 \pm 31$ & $69 \pm 24$ & $115 \pm 37$ \\
\hline V388 Peg & $15 \mathrm{~A}-33 \mathrm{~A}$ & $59 \pm 22$ & $46 \pm 15$ & $55 \pm 16$ \\
\hline V388 Peg & $15 \mathrm{~A}-33 \mathrm{~B}$ & $32 \pm 14$ & $26 \pm 10$ & $71 \pm 19$ \\
\hline FO Aqr & 13B-19A & $46 \pm 19$ & $62 \pm 22$ & $126 \pm 36$ \\
\hline FO Aqr & 13B-19B & $40 \pm 20$ & $46 \pm 19$ & $96 \pm 29$ \\
\hline Aqr 2 & $15 \mathrm{~A}-33 \mathrm{~A}$ & $38 \pm 17$ & $39 \pm 13$ & $64 \pm 16$ \\
\hline Aqr 2 & $15 \mathrm{~A}-33 \mathrm{~B}$ & $37 \pm 14$ & $28 \pm 13$ & $64 \pm 22$ \\
\hline AO Psc & 13B-19A & $87 \pm 25$ & $69 \pm 34$ & $117 \pm 41$ \\
\hline AO Psc & 13B-19B & $73 \pm 29$ & $62 \pm 23$ & $107 \pm 31$ \\
\hline Aqr 5 & $15 \mathrm{~A}-34 \mathrm{~A}$ & $44 \pm 13$ & $40 \pm 11$ & $64 \pm 18$ \\
\hline Aqr 5 & $15 \mathrm{~A}-34 \mathrm{~B}$ & $23 \pm 13$ & $36 \pm 13$ & $93 \pm 23$ \\
\hline BW Scl & $15 \mathrm{~A}-34 \mathrm{~A}$ & $42 \pm 16$ & $30 \pm 14$ & $111 \pm 36$ \\
\hline
\end{tabular}


JVLA MCV SuRvey Data

Table 4 (continued)

\begin{tabular}{lrrrr}
\hline \hline GCVS Name & Semester & $\begin{array}{c}\text { C Flux } \\
\text { Density } \\
(\mu \text { Jy/beam })\end{array}$ & $\begin{array}{c}\text { X Flux } \\
\text { Density } \\
(\mu \text { Jy/beam })\end{array}$ & $\begin{array}{c}\text { K Flux } \\
\text { Density } \\
(\mu \text { Jy } / \text { beam })\end{array}$ \\
\hline BW Scl & 15A-34B & $34 \pm 16$ & $30 \pm 10$ & $142 \pm 44$ \\
HU Aqr & 13B-19C & $70 \pm 43$ & $198 \pm 82$ & $214 \pm 55$ \\
HU Aqr & 13B-19D & $206 \pm 76$ & $191 \pm 91$ & $143 \pm 45$ \\
FO Aqr & 13B-19C & $72 \pm 30$ & $77 \pm 27$ & $177 \pm 54$ \\
FO Aqr & 13B-19D & $81 \pm 31$ & $99 \pm 29$ & $136 \pm 43$ \\
AO Psc & 13B-19C & $39 \pm 46$ & $78 \pm 37$ & $153 \pm 54$ \\
AO Psc & 13B-19D & $85 \pm 42$ & $57 \pm 24$ & $146 \pm 47$ \\
\hline
\end{tabular}

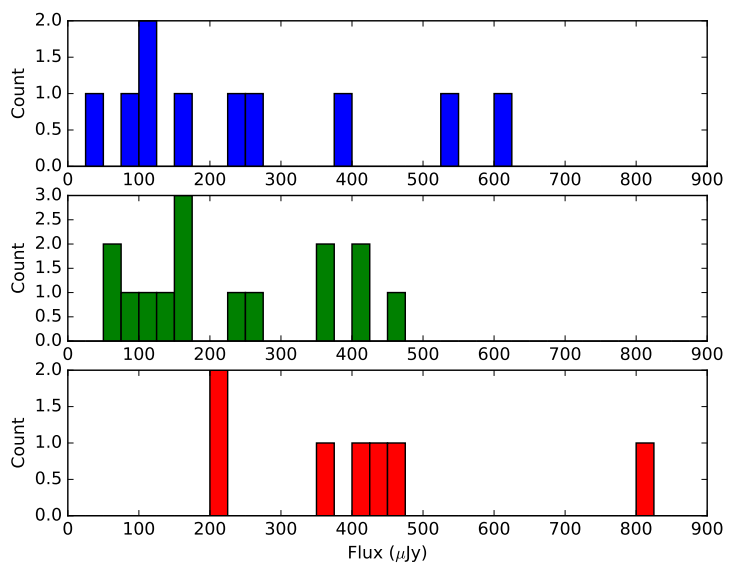

Figure 1. Flux histograms. From top to bottom, the panels are the C-band, X-band, and K-band flux densities, respectively.

The flux densities of AE Aqr, being > $5 \mathrm{mJy}$, are excluded.

\section{DISCUSSION}

There are currently eleven known CVs that are radio sources: four are polars, AM Her, AR UMa, ST LMi, and V834 Cen; four are IPs or DQ Her stars, AE Aqr, BG CMi, DQ Her, and GK Per; and three are considered non-magnetic CVs: RW Sex, TT Ari, and V603 Aql, which are classified as dwarf novae or nova-likes. This survey increases the number of $\mathrm{CVs}$ that are radio sources to 25. The significance of all detections except one (BS Tri) are $(>5 \sigma)$. This result is a twofold increase in the number of CVs that are radio sources. Fifteen of the new detections are polars; three are IPs, and one is a non-magnetic $\mathrm{CV}$.

The radio fluxes of most detections are in the range 47-470 $\mu \mathrm{Jy}$, except for AE Aqr, AM Her, AR UMa, and MR Ser (see Figure 1). AE Aqr and AR UMa show radio emission at all frequencies (C-, X-, \& K-bands) and at both epochs. Whereas AM Her shows emission at all frequencies at only a single epoch. The remaining sixteen sources show emission at no more than two frequencies. All CVs show variable emission at some level, even the three strong sources; AE Aqr, AM Her, and AR UMa. The variability of the radio flux at different epochs and frequencies is characteristic of short timescale flares. For example, the polar AM Her shows faint $(114 \mu \mathrm{Jy}) \mathrm{C}$-band emission during the first observation on 2013-12-02 and moderate emission (154, 243, \& $818 \mu \mathrm{Jy})$ at three frequencies $(\mathrm{C}, \mathrm{X}, \& \mathrm{~K}$, respectively) during the second observation 34 days later on 2014-01-06.

The small sample (19) of detections limits the comparison of the frequency dependence of the radio emission to two subclasses, polars and non-polars. For the polars, the number of detections at the $\mathrm{C}-$, $\mathrm{X}$-, and $\mathrm{K}$-bands are 7,12 , and 7 , respectively. For the non-polars with the exclusion of AE Aqr, the numbers are respectively 3, 3 , and 0 . This suggests that the polars are more likely to emit at higher frequencies $(>22 \mathrm{GHz}$ ) compared to the non-polars. We note that, except for AE Aqr, these observations are the first detections of polars at $22 \mathrm{GHz}$.

A notable feature of Table 3 is the fourteen detections that show essentially $100 \%$ circularly polarized emission (i.e., a source is only seen in the left-hand or righthand circular polarization image), whereas the remaining five detections show no (linear and circularly) polarized emission. In addition the timescale of the emission is short, $<5$ minutes. These two properties are characteristic of electron-cyclotron maser emission like that of the ten minute flare of AM Her. Four of these sources show polarized emission at $22 \mathrm{GHz}$. The other five radio sources show no (or weakly) polarized emission. Three of them are the well-known MCVs, AM Her, AR UMa, and AE Aqr. These results suggest that MCVs might 
be divided into two classes of radio emitters: emission dominated by weakly polarized gyrosynchrotron radiation and by highly polarized electron-cyclotron maser emission.

\section{CONCLUSIONS}

The current survey indicates that MCVs are highly variable radio sources that can emit at frequencies as high as $22 \mathrm{GHz}$ via gyrosynchrotron and electroncyclotron maser emission. The data suggest that the polars are more likely to emit at higher frequencies than the non-polars, and MCVs can be divided into two classes of radio emitters: those dominated by weakly polarized gyrosynchrotron emission and those by highly polarized electron-cyclotron maser emission.
Paper two of this survey presents a more in-depth analysis of these data by investigating the two radiation mechanisms and the possibility of two classes of radio emitting MCVs. Paper three of this survey will present high-resolution VLBI observations of MCVs, including the sources detected in this paper.

P.E.B. and P.A.M. acknowledge the guidance and inspiration of their late mentor and colleague G. Chanmugam, who began the search for radio emission from magnetic cataclysmic variables over 35 years ago. The National Radio Astronomy Observatory is a facility of the National Science Foundation operated under cooperative agreement by Associated Universities, Inc. This research has made use of the SIMBAD database, operated at CDS, Strasbourg, France (Wenger et al. 2000).

\section{REFERENCES}

Bastian, T. S. 1987, PhD Thesis, Univ. Colorado

Beasley, A. J., Bastian, T. S., Ball, L., \& Wu, K. 1994, AJ, 108, 2207

Bookbinder, J. A., \& Lamb, D. Q. 1987, ApJ, 323, 131

Chanmugam, G., \& Wagner, R. L. 1978, ApJ, 222, 641

Chanmugam, G., \& Dulk, G. A. 1982, ApJ, 255, L107

Chanmugam, G. 1987, Ap\&SS, 130, 53

Condon, J. J., Cotton, W. D., Fomalont, E. B., Kellermann, K. I., Miller, N., Perley, R. A., Scott, D., Vernstrom, T., \& Wall, J. V. ApJ, 758, 23

Coppejans, D. L., Körding, E. G., Miller-Jones, J. C. A., Rupen, M. P., Knigge, C., Sivakoff, G. R., \& Groot, P. J. 2015, MNRAS, 451, 3801

Coppejans, D. L., Körding, E. G., Miller-Jones, J. C. A., Rupen, M. P., Sivakoff, G. R., Knigge, C., Groot, P. J., Woudt, P. A., Waagen, E. O., \& Templeton, M. 2016, MNRAS, 463, 2229

Johns-Krull, C. M., \& Valenti, J. A. 1996, ApJ, 459, L95

Dulk, G. A., Bastian, T. S., \& Chanmugam, G. 1983, ApJ, 273, 249
Goldreich, P., \& Lynden-Bell, D. 1969, ApJ, 156, 59

Hjellming, R. M. \& Wade, C. M. 1970, ApJL, 162, 1

Mason, P. A., Fisher, P. L., \& Chanmugam, G. 1996, A\&A, 310, 132

Mason, P. A. \& Gray, C. L. 2007, ApJ, 600, 662

Pavelin, P. E., Spencer, R. E., \& Davis, R. J. 1994, MNRAS, 269, 779

Rappaport, S., Verbunt, F., \& Joss, P.C. 1983, ApJ, 275, 713

Spruit, H. C. \& Ritter, H. 1983, A\&A, 124, 267

Wenger, M., Ochsenbein, F., Egret, D., Dubois, P., Bonnarel, F., Borde, S., Genova, F., Jasniewicz, G., Laloë, S., Lesteven, S., \& Monier, R. 2000, A\&AS, 143, 9

Wright, A. E., Cropper, M., Stewart, R. T., Nelson, G. J., \& Slee, O. B. 1988, MNRAS, 231, 319 